TRANSACTIONS OF THE

AMERICAN MATHEMATICAL SOCIETY

Volume 358, Number 4, Pages 1715-1740

S 0002-9947(05)03793-1

Article electronically published on November 1, 2005

\title{
MULTIPLE HOMOCLINIC ORBITS IN CONSERVATIVE AND REVERSIBLE SYSTEMS
}

\author{
ALE JAN HOMBURG AND JÜRGEN KNOBLOCH
}

\begin{abstract}
We study dynamics near multiple homoclinic orbits to saddles in conservative and reversible flows. We consider the existence of two homoclinic orbits in the bellows configuration, where the homoclinic orbits approach the equilibrium along the same direction for positive and negative times.

In conservative systems one finds one parameter families of suspended horseshoes, parameterized by the level of the first integral. A somewhat similar picture occurs in reversible systems, with two homoclinic orbits that are both symmetric. The lack of a first integral implies that complete horseshoes do not exist. We provide a description of orbits that necessarily do exist.

A second possible configuration in reversible systems occurs if a non-symmetric homoclinic orbit exists and forms a bellows together with its symmetric image. We describe the nonwandering set in an unfolding. The nonwandering set is shown to simultaneously contain one-parameter families of periodic orbits, hyperbolic periodic orbits of different index, and heteroclinic cycles between these periodic orbits.
\end{abstract}

\section{INTRODUCTION}

Consider a conservative vector field possessing a hyperbolic equilibrium $p$ so that the linearized vector field at $p$ has real weakest stable and unstable eigenvalues. Homoclinic orbits to such an equilibrium occur persistently, under certain transversality conditions. Furthermore, there is a sheet of periodic solutions accumulating onto the homoclinic orbit VanFie92. Consider a reversible vector field with a symmetric hyperbolic equilibrium $p$ as above. Under natural transversality conditions, symmetric homoclinic orbits to $p$ are persistent and have attached to them a sheet of periodic solutions Dev77, VanFie92. So far there are no essential differences between dynamics near homoclinic orbits in conservative systems and near symmetric homoclinic orbits in reversible systems. Of course, in reversible systems one can also find non-symmetric homoclinic orbits. Whereas symmetric homoclinic orbits persist under perturbations, non-symmetric homoclinic orbits are expected to break under perturbations. Unfoldings require one-parameter families of vector fields.

In both conservative and reversible systems, there can be more than one homoclinic orbit to $p$. To fix thoughts, consider two different homoclinic orbits to $p$. The nontrivial case, which we will consider, is where both homoclinic orbits approach $p$ from the same direction, both for positive and negative times (one needs at least a four-dimensional state space for this to be possible). Following AroGolKru91, we

Received by the editors September 19, 2002 and, in revised form, July 1, 2004.

2000 Mathematics Subject Classification. Primary 34Cxx, 37Cxx, 37Gxx. 
refer to the union of two such homoclinic orbits as a homoclinic bellows. In predating Russian literature TurShi87, Tur88, the bellows configuration was referred to as a homoclinic figure eight. Bifurcation diagrams for two-parameter unfoldings of a homoclinic bellows in general systems, without symmetry assumptions, are provided in TurShi87, Tur88. In Hom93, the nonwandering set near homoclinic bellows in systems with $\mathbb{Z}_{2}$-symmetry has been studied.

We will study the dynamics near bellows in conservative and reversible systems. In reversible systems, assuming that $p$ is a symmetric equilibrium, two different possibilities occur. In one case there are two homoclinic orbits $\Gamma_{1}, \Gamma_{2}$ to $p$ that are both symmetric under the involution $R$ that provides the reversibility: $\Gamma_{1}=R\left(\Gamma_{1}\right)$ and $\Gamma_{2}=R\left(\Gamma_{2}\right)$. A second possible configuration in reversible systems occurs if a non-symmetrical homoclinic orbit $\Gamma_{1}$ together with its symmetric image $\Gamma_{2}=R\left(\Gamma_{1}\right)$ forms a homoclinic bellows.

In all cases the analysis is enabled by the construction of a collection of center manifolds, parameterized by the bi-infinite symbol space $\mathcal{B}_{2}$ on two symbols (Theorem 2.1). This reduces the dynamics to a skew product system of interval maps over the base space $\mathcal{B}_{2}$ on which the shift operator acts. For reversible systems, one finds a reversibility for the skew product system as well.

The dynamics near homoclinic bellows in conservative systems and near bellows of symmetric homoclinic orbits in reversible systems, has similar features, but shows differences also. In conservative systems, there is a one-parameter family of suspended Smale horseshoes near the union of the two homoclinic orbits, parameterized by levels of the first integral (Theorem 3.2). Near a bellows of symmetric homoclinic orbits in reversible systems there is still an infinite nonwandering set, corresponding to an uncountable number of symbolic sequences, but in general there is not a complete family of suspended horseshoes (Theorem 4.2 and Theorem 4.3). The nonexistence of a first integral allows for a drift in the dynamics on the center manifolds.

A different scenario occurs in reversible systems, for homoclinic bellows consisting of non-symmetric homoclinic orbits. One-parameter families of vector fields are considered to unfold the homoclinic bifurcations. This situation has no analogue in conservative systems, where homoclinic orbits persist under perturbations. An analysis of the dynamics on the center manifolds reveals a surprising complexity (Theorem 5.1] and Theorem [5.2).

The comparison between conservative (in particular, Hamiltonian) vector fields and reversible vector fields is particularly interesting, since vector fields can be both conservative (Hamiltonian) and reversible, and perturbations can destroy one of these structures. Several models are known that are both Hamiltonian and reversible, and possess a homoclinic bellows. Let us indicate one example from the study of solitary waves in a fifth order equation for gravity-capillary water waves ChaGro97, WagCha02,

$$
u_{t}+\frac{2}{15} u_{x x x x x}-b u_{x x x}+3 u u_{x}+2 u_{x} u_{x x}+u u_{x x x}=0 .
$$

Making a traveling wave ansatz $u(x, t)=v(x-a t)$ and integrating the resulting ordinary differential equation once, one obtains the fourth order equation

$$
\frac{2}{15} v^{i v}-b v^{\prime \prime}+a v+\frac{3}{2} v^{2}-\frac{1}{2}\left(v^{\prime}\right)^{2}+\left[v v^{\prime}\right]^{\prime}=0 .
$$


As a first order system in the variables $v, v^{\prime}, v^{\prime \prime}, v^{\prime \prime \prime}$, this system is Hamiltonian and reversible with respect to $R\left(v, v^{\prime}, v^{\prime \prime}, v^{\prime \prime \prime}\right)=\left(v,-v^{\prime}, v^{\prime \prime},-v^{\prime \prime \prime}\right)$. There is evidence (analytical, partly backed up by numerical computations), that for certain values of the parameters $a, b$ close to $a=0, b=2$, the system possesses a homoclinic bellows WagCha02. Bellows are created in this system from a bifurcation of a homoclinic orbit to a non-hyperbolic equilibrium with a double zero eigenvalue. WagCha02 describes more applications whose study involves this bifurcation.

In a different set of scenarios, homoclinic bellows bifurcate from degenerate homoclinic orbits FieTur96, Kno97, Van92. Then both homoclinic orbits forming the bellows arise simultaneously and are almost copies of each other. Homoclinic bellows can also be found in systems with an orbit flip bifurcation of a homoclinic orbit SanJonAle97, Tur01. Homoclinic bellows consisting of non-symmetric homoclinic orbits appear in a reversible homoclinic pitchfork bifurcation from a degenerate homoclinic orbit [Kno97.

\section{Center manifolds near a homoclinic Bellows}

In this section we construct center manifolds near homoclinic bellows. Although we apply the constructions for systems with a special structure, namely the existence of a first integral or reversibility, we will consider general vector fields. The notation introduced below will be used in following sections as well. The proofs of the main results, Theorem 2.1 and Theorem 2.2. are postponed to Appendix C. The proof relies on normal form theory and Shil'nikov coordinates, developed in Appendix A. Connections with Lin's method for deriving bifurcation equations in a Lyapunov-Schmidt type approach, are explained in Appendix B.

Let $X$ be a smooth vector field on a finite dimensional Riemannian manifold $M$. Assume that $X$ possesses a hyperbolic equilibrium $p$, and that $D X(p)$ has eigenvalues $\left\{\lambda_{i}^{s s}\right\}_{1 \leq i \leq q_{s s}}, \lambda^{s}, \lambda^{u},\left\{\lambda_{i}^{u u}\right\}_{1 \leq i \leq q_{u u}}$ with

$$
\operatorname{Re}\left(\lambda_{i}^{s s}\right)<\lambda^{s}<0<\lambda^{u}<\operatorname{Re}\left(\lambda_{i}^{u u}\right) .
$$

The eigenvalues $\lambda_{i}^{s s}$ are called the strong stable eigenvalues, $\lambda^{s}$ is the weak stable eigenvalue. Similarly, $\lambda^{u}$ is the weak unstable eigenvalue and $\lambda_{i}^{u u}$ are the strong unstable eigenvalues. Let $T_{p} M=E^{s s} \oplus E^{s} \oplus E^{u} \oplus E^{u u}$ be the resulting $D X(p)$ invariant splitting of $T_{p} M$.

Recall that a homoclinic orbit (to $p$ ) is a solution of $\dot{x}=X(x)$ converging to $p$ for $t \rightarrow \pm \infty$. Therefore a homoclinic orbit lies in the intersection of the stable and unstable manifold $W^{s s, s}(p)$ and $W^{u, u u}(p)$ of $p$. Assume that $X$ has two homoclinic orbits $\Gamma_{1}, \Gamma_{2}$ forming a bellows:

$$
\begin{array}{r}
e^{s}=\lim _{t \rightarrow \infty} \dot{\Gamma}_{1}(t) /\left\|\dot{\Gamma}_{1}(t)\right\|=\lim _{t \rightarrow \infty} \dot{\Gamma}_{2}(t) /\left\|\dot{\Gamma}_{2}(t)\right\| \in E^{s}, \\
e^{u}=\lim _{t \rightarrow-\infty} \dot{\Gamma}_{1}(t) /\left\|\dot{\Gamma}_{1}(t)\right\|=\lim _{t \rightarrow-\infty} \dot{\Gamma}_{2}(t) /\left\|\dot{\Gamma}_{2}(t)\right\| \in E^{u} .
\end{array}
$$

This implies that

$$
\Gamma_{i} \not \subset W^{s s}(p), \quad \Gamma_{i} \not \subset W^{u u}(p), \quad i=1,2,
$$

where $W^{s s}(p)$ is the $q_{s s}$-dimensional strong stable manifold of $p$ and $W^{u u}(p)$ is the $q_{u u}$-dimensional strong unstable manifold of $p$. Orbit-flips are thus excluded Yan87, San93. Besides the stable and unstable manifolds there exist center unstable manifolds $W^{s, u, u u}(p)$ with tangent space $E^{s} \oplus E^{u} \oplus E^{u u}$ at $p$ and center stable 
manifolds $W^{s s, s, u}(p)$ with tangent space $E^{s s} \oplus E^{s} \oplus E^{u}$ at $p$. Assume the following transversality conditions for the homoclinic bellows:

$$
W^{s s, s, u}(p) \pitchfork_{\Gamma_{1} \cup \Gamma_{2}} W^{u, u u}(p), \quad W^{s, u, u u}(p) \pitchfork_{\Gamma_{1} \cup \Gamma_{2}} W^{s s, s}(p) .
$$

This condition excludes the inclination flip situation Yan87, Den93, KisKokOka93, HomKokKru94. It also implies that both $\Gamma_{1}$ and $\Gamma_{2}$ are non-degenerate: along $\Gamma_{i}$, $i=1,2$, the tangent spaces of $W^{s s, s}(p)$ and $W^{u, u u}(p)$ intersect only in the vector field direction.

There are smooth vector bundles $F^{s s, s, u}$ and $F^{s s}$ along the stable manifold $W^{s s, s}(p)$, invariant for the flow of $D X$, with $F_{p}^{s s, s, u}=E^{s s} \oplus E^{s} \oplus E^{u}$ and $F_{p}^{s s}=E^{s s}$. Similarly, there are smooth vector bundles $F^{s, u, u u}$ and $F^{u u}$ along the unstable manifold $W^{u, u u}(p)$, with $F_{p}^{s, u, u u}=E^{s} \oplus E^{u} \oplus E^{u u}$ and $F_{p}^{u u}=E^{u u}$. By the transversality assumptions (3) and (4), $F^{s s}, F^{u u}, F^{s s, s, u}$ and $F^{s, u, u u}$ extend to continuous vector bundles along $\{p\} \cup \Gamma_{1} \cup \Gamma_{2}$; see e.g. Hom96. The intersection of $F^{s s, s, u}$ and $F^{s, u, u u}$ provides a continuous bundle $F^{s, u}$ along $\{p\} \cup \Gamma_{1} \cup \Gamma_{2}$ with $F_{p}^{s, u}=E^{s} \oplus E^{u}$. We will assume that

$$
F^{s, u} \text { is an orientable bundle along }\{p\} \cup \Gamma_{1} \cup \Gamma_{2} .
$$

For symmetric homoclinic orbits in reversible systems and for homoclinic orbits in conservative systems, this condition will turn out to be automatically fulfilled.

Let $\Sigma_{1}$ and $\Sigma_{2}$ be cross-sections of $\Gamma_{1}$ and $\Gamma_{2}$, respectively. Write $\gamma_{i}=\Gamma_{i} \cap \Sigma_{i}$. By $\Psi: \Sigma_{1} \cup \Sigma_{2} \rightarrow \Sigma_{1} \cup \Sigma_{2}$ we denote the first return map defined on a subset of $\Sigma_{1} \cup \Sigma_{2}$. Associated to an orbit $x=\{x(i)\}, x(i+1)=\Psi(x(i))$ for $i \in \mathbb{Z}$, in the nonwandering set $\Omega$ of $\Psi$, there is an itinerary $\Upsilon(x): \mathbb{Z} \rightarrow\{1,2\}$ defined by

$$
\Upsilon(x)(i)=j, \text { if } x(i) \in \Sigma_{j} .
$$

Let $\mathcal{B}_{2}$ be the set of itineraries $\mathbb{Z} \rightarrow\{1,2\}$, equipped with the product topology. The shift operator $\sigma: \mathcal{B}_{2} \rightarrow \mathcal{B}_{2}$ is, as usual, given by $\sigma(y)(k)=y(k+1)$.

The following theorem shows that the nonwandering set of $\Psi$ is contained in a Cantor bouquet of center manifolds, bounded by the bellows. The center manifolds are tangent to the bundle $F^{s, u}$ along the homoclinic orbits. In the following sections we discuss the dynamics within this Cantor bouquet of center manifolds, for bellows in reversible and conservative systems.

Theorem 2.1. For each $\eta \in \mathcal{B}_{2}$, there is a one-dimensional normally hyperbolic center manifold $W_{\eta}^{c}$ for $\Psi$, so that any orbit $x$ with itinerary $\Upsilon(x)=\eta$, satisfies $x(0) \in W_{\eta}^{c}$. The manifold $W_{\eta}^{c}$ is continuously differentiable and depends continuously on $\eta$. It contains $\gamma_{\eta(0)}$, and the tangent space of $W_{\eta}^{c}$ here equals $F_{\gamma_{\eta(0)}}^{s, u} \cap T_{\gamma_{\eta(0)}} \Sigma_{\eta(0)}$. Moreover, $W_{\sigma(\eta)}^{c}=\Psi\left(W_{\eta}^{c}\right)$.

We postpone the proof of this theorem to Appendix C] See Hom96, RouRou96, ShaTur99, ChoLiuYi00, San00 for the construction of center manifolds near homoclinic orbits (i.e. with $\eta$ equal to a constant sequence of 1's or 2's).

Note that, if $x \in W_{\eta}^{c}$, then either the orbit of $x$ leaves a small neighborhood $\mathcal{U}$ of $\Gamma_{1} \cup \Gamma_{2} \cup\{p\}$, or $\Upsilon(x)=\eta$. Assume that $\eta$ is a recurrent point for $\sigma$ in $\mathcal{B}_{2}$. Then, if the orbit of $x \in W_{\eta}^{c}$ stays in $\mathcal{U}$ but iterates of $x$ do not converge to $\Gamma_{1} \cup \Gamma_{2} \cup\{p\}$, there exists a nonwandering point for $\Psi$ with $\eta$ as its itinerary.

In our study of non-symmetric homoclinic orbits in reversible systems, we will need to consider families of vector fields that unfold homoclinic bifurcations. Let 
$\left\{X_{\mu}\right\}$ be a smooth family of vector fields with $X_{0}=X$. Write $\Psi_{\mu}$ for the return map for $X_{\mu}$ on $\Sigma_{1} \cup \Sigma_{2}$.

Theorem 2.2. For each small $\mu$ and each $\eta \in \mathcal{B}_{2}$, there is a one-dimensional normally hyperbolic center manifold $W_{\eta}^{c}$ for $\Psi_{\mu}$, so that any orbit $x$ with itinerary $\Upsilon(x)=\eta$, satisfies $x(0) \in W_{\eta}^{c}$. The manifold $W_{\eta}^{c}$ is continuously differentiable, depends differentiably on $\mu$, and depends continuously on $\eta$. It satisfies $W_{\sigma(\eta)}^{c}=$ $\Psi_{\mu}\left(W_{\eta}^{c}\right)$.

\section{Conservative systems}

Consider a vector field $X$ on a finite dimensional manifold $M$ which is conservative. We assume that

there is a smooth mapping $H: M \rightarrow \mathbb{R}$ such that $D H(x) X(x)=0, \forall x \in M$.

Suppose that $X$ has a hyperbolic equilibrium $p$ and that the principal stable and unstable eigenvalues of $D X(p)$ are real and simple (as assumed in Section 2). We assume that there are two homoclinic orbits $\Gamma_{1}$ and $\Gamma_{2}$ asymptotic to $p$ forming a bellows; so (11) and (2) hold. Finally, we assume the transversality condition (4) and that

$$
D H \text { is non-singular along both } \Gamma_{1} \text { and } \Gamma_{2} \text {. }
$$

It is sufficient to require that $D H$ is non-singular in one point on $\Gamma_{1}$ and $\Gamma_{2}$. Then we know

Theorem 3.1 (VanFie92]). Both homoclinic orbits $\Gamma_{1}$ and $\Gamma_{2}$ are accompanied by a family of 1-periodic orbits. More precisely, there is a number $\tilde{\tau}>0$ such that for each $\tau>\tilde{\tau}$ there are two smooth families $y_{\tau}^{1}$ and $y_{\tau}^{2}$ of 1-periodic orbits with period $\tau$ such that $y_{\tau}^{i} \rightarrow \Gamma_{i}$ as $\tau \rightarrow \infty, i=1,2$.

As usual we call a periodic orbit 1-periodic with respect to a given homoclinic orbit $\Gamma$ if it goes just once around $\Gamma$. Theorem 3.1 implies that the orbits $\Gamma_{1}$ and $\Gamma_{2}$ are non-twisted, that is, (5) holds. Twisted homoclinic orbits have a twodimensional non-orientable center manifold and hence they cannot have 1-periodic orbits in an arbitrarily small neighborhood. Further the existence of the families of 1-periodic orbits implies that the principal eigenvalues are in resonance: $\lambda^{s}=-\lambda^{u}$. A way to see this is by considering the equation for detecting 1-periodic orbits; see Appendix B. For that we put $x_{u}=x_{u}(j+1)=x_{u}(j)$ in equation (49). It follows that $\beta=1$, which means $\lambda^{s}=-\lambda^{u}$. Finally, the bellows configuration implies $\operatorname{sign} D_{\tau} H\left(y_{\tau}^{1}\right)=\operatorname{sign} D_{\tau} H\left(y_{\tau}^{2}\right)$. This follows since the periodic orbits $y_{\tau}^{1}$ and $y_{\tau}^{2}$ are confined to orientable center manifolds which are tangent to each other at $p$. The sheets of periodic orbits are transverse to the level sets of $H$, since $D H$ is nonsingular along the homoclinic orbits. We refer to Figure 1 for an illustration.

So in each level set for $H$ on one side of $H(p)$ there are two hyperbolic periodic orbits whose stable and unstable manifolds have the chance to intersect transversally. Consequently, one can expect nontrivial dynamics within level sets. Indeed Theorem 3.2 gives a one-parameter family of hyperbolic horseshoes.

Theorem 3.2. Let $\{p\} \cup \Gamma_{1} \cup \Gamma_{2}$ be a bellows of a conservative vector field $X$ with properties as stated above. Then, in a small neighborhood $\mathcal{U}$ of the bellows there exists a one-parameter family of hyperbolic horseshoes, parameterized by the first integral $H$, for values on one side of $H(p)$. 


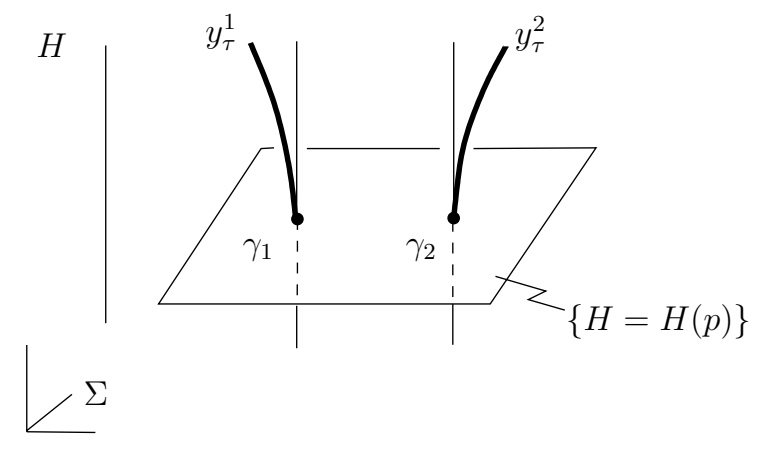

FiguRe 1. Families of 1-periodic orbits attached at a homoclinic bellows in conservative systems, depicted in a single cross-section $\Sigma$ close to $p$.

Proof. Let $\Sigma_{1}$ and $\Sigma_{2}$ be cross-sections of $\Gamma_{1}$ and $\Gamma_{2}$, respectively. The corresponding coordinates (see Section $\mathbb{\mathrm { A }}$ ), we denote by $\left(x_{s s}^{i}, x_{u}^{i}, x_{u u}^{i}\right), i=1,2$. For a given itinerary $\eta$, the center manifold $W_{\eta}^{c}$ can be parameterized by $x_{u}^{\eta(0)}$. There is a mapping $w_{\eta}: \mathbb{R} \rightarrow \Sigma_{\eta(0)}$ with $W_{\eta}^{c}=w_{\eta}(\mathbb{R})$. For all small positive values of $x_{u}$, $w_{\eta}\left(x_{u}\right)$ is in the domain of the return map $\Psi$.

Further, the coordinate lines of $x_{u}^{i}, i=1,2$, are transverse to the level set $\{H=H(p)\}$. We may assume that $x_{u}^{i}>0$ corresponds to $H>H(p)$. Then there is a $\tilde{\delta}>0$ such that for all $\eta \in \mathcal{B}_{2}$ and for all $H(p)<H^{*}<H(p)+\tilde{\delta}$, there is a $x_{u}<\delta$ such that $w_{\eta}\left(x_{u}\right) \in\left\{H=H^{*}\right\}$.

Then the existence of a one-parameter family of horseshoes follows from Theorem 2.1 noting that the center manifolds intersect the level sets transversally and that level sets of the first integral are invariant. Hyperbolicity of the horseshoes within level sets follows from the normal hyperbolicity of the center manifolds.

Consider, in particular, a Hamiltonian vector field $X$ (on an even dimensional symplectic manifold). Then by the "Nonintegrability theorem" presented in KolLer96 this vector field is nonintegrable. This follows from the existence of periodic orbits of saddle type (within a level set) and transversal (again within level sets) homoclinic orbits to those periodic orbits.

\section{Symmetric Homoclinic orbits In REVERSible SyStemS}

Consider a vector field $X$ on a $2 n$-dimensional Riemannian manifold $M$ that is reversible with respect to an involution $R$ :

$$
D R(x) X(x)=-X \circ R(x) .
$$

Let us denote the flow of the vector field $X$ by $\left\{\phi^{t}\right\}_{t \in \mathbb{R}}$. Then, due to the reversibility,

$$
\phi^{t} \circ R=R \circ \phi^{-t} .
$$

Suppose that $X$ has a symmetric hyperbolic equilibrium $p \in$ Fix $R$ and that $D X(p)$ has eigenvalues $\left\{\lambda_{i}^{s s}\right\}_{1 \leq i \leq n-1}, \lambda^{s}, \lambda^{u}=-\lambda^{s},\left\{\lambda_{i}^{u u}=-\lambda_{i}^{s s}\right\}_{1 \leq i \leq n-1}$ with

$$
\operatorname{Re}\left(\lambda_{i}^{s s}\right)<\lambda^{s}<0 .
$$


The existence of a symmetric hyperbolic fixed point $p$ enforces an even dimensional state space, because the involution $R$ maps the stable manifold of $p, W^{s s, s}(p)$, onto the unstable manifold $W^{u, u u}(p)$. Also, the strong stable and strong unstable manifolds are $R$-images of each other. Consequently, the subspaces $E^{s s}, E^{u u}$ and $E^{s}, E^{u}$, respectively, will be mapped onto each other by $D R(p)$. Furthermore, the space Fix $D R(p)$ is $n$-dimensional. Therefore (at least locally around $p$ ), the manifold Fix $R$ is $n$-dimensional. Also the resonance of the eigenvalues $\lambda^{s}=-\lambda^{u}$ is a consequence of the reversibility.

An orbit $\mathfrak{o}$ is called symmetric if $R(\mathfrak{o})=\mathfrak{o}$. Each symmetric orbit $\mathfrak{o}$ intersects Fix $R$ at least once but at most twice. The latter case happens iff $\mathfrak{o}$ is periodic.

Suppose $X$ has two different symmetric homoclinic orbits $\Gamma_{1}, \Gamma_{2}$ forming a bellows. Note that in this case (2) follows from (11). Although the manifolds $W^{s, u, u u}(p)$ and $W^{s s, s, u}(p)$ are not uniquely defined they can be chosen such that they are $R$ images of each other. We assume the transversality condition (4). Condition (4) implies that $W^{s s, s}(p)$ intersects Fix $R$ transversally (because the homoclinic orbits are non-degenerate). Hence the homoclinic orbits $\Gamma_{i}, i=1,2$ persist under perturbations of the vector field.

In the present situation there holds an assertion similar to Theorem 3.1 .

Theorem 4.1 ([VanFie92]). Both homoclinic orbits $\Gamma_{1}$ and $\Gamma_{2}$ are accompanied by a family of symmetric 1-periodic orbits. More precisely, there is a number $\tilde{\tau}>0$ such that for each $\tau>\tilde{\tau}$ there are two smooth families $y_{\tau}^{1}$ and $y_{\tau}^{2}$ of symmetric 1-periodic orbits with period $\tau$ such that $y_{\tau}^{i} \rightarrow \Gamma_{i}$ as $\tau \rightarrow \infty, i=1,2$.

As in the conservative case this theorem implies that the orbits $\Gamma_{1}$ and $\Gamma_{2}$ are non-twisted; so condition (5) is fulfilled automatically. Up to this stage the situation resembles the one in the conservative case (if we regard symmetric orbits as the natural counterpart of orbits in conservative systems).

Write $\gamma_{i}=\Gamma_{i} \cap$ Fix $R$. Due to reversibility, $F_{R x}^{u u}=D R(x) F_{x}^{s s}$, for all $x \in \Gamma_{1} \cup \Gamma_{2}$. In particular, $F_{\gamma_{i}}^{u u}=D R\left(\gamma_{i}\right) F_{\gamma_{i}}^{s s}$. This and (4) show that $T_{\gamma_{i}} M$ can be represented as

$$
T_{\gamma_{i}} M=F_{\gamma_{i}}^{u u} \oplus F_{\gamma_{i}}^{s s} \oplus F_{\gamma_{i}}^{+} \oplus F_{\gamma_{i}}^{-}
$$

where $F_{\gamma_{i}}^{+} \subset$ Fix $\left(D R\left(\gamma_{i}\right)\right)$ and $F_{\gamma_{i}}^{-} \subset$ Fix $\left(-D R\left(\gamma_{i}\right)\right)$. In particular, $F_{\gamma_{i}}^{-}$can be chosen as $F_{\gamma_{i}}^{-}=\operatorname{span}\left(X\left(\gamma_{i}\right)\right)$, since $X\left(\gamma_{i}\right) \in$ Fix $\left(-D R\left(\gamma_{i}\right)\right)$. With that we define cross-sections $\Sigma_{i}$ of $\Gamma_{i}$ as follows: Let $U_{i}$ be an R-symmetric open neighborhood of $\gamma_{i}$ and let $\left(\tilde{\rho}_{i}, U_{i}\right)$ be a chart, $\tilde{\rho}_{i}: U_{i} \subset M \rightarrow T_{\gamma_{i}} M$. Then $\rho_{i}$ given by $\rho_{i}(x)=$ $\frac{1}{2}\left(\tilde{\rho}_{i}(x)+D R\left(\gamma_{i}\right) \tilde{\rho}_{i}(R x)\right)$ is a symmetric map:

$$
D R\left(\gamma_{i}\right) \rho_{i}(x)=\rho_{i}(R x)
$$

for all $x \in U_{i}$. Define $\Sigma_{i}$ by

$$
\Sigma_{i}=\rho_{i}^{-1}\left(F_{\gamma_{i}}^{u u} \oplus F_{\gamma_{i}}^{s s} \oplus F_{\gamma_{i}}^{+}\right)
$$

By construction $\Sigma_{1}$ and $\Sigma_{2}$ are symmetric cross-sections: $R\left(\Sigma_{1}\right)=\Sigma_{1}$ and $R\left(\Sigma_{2}\right)=$ $\Sigma_{2}$. The cross-sections $\Sigma_{i}$ contain Fix $R$ : $U_{i} \cap$ Fix $R \subset \Sigma_{i}, i=1,2$. By (7), $R$ acts linearly on $\Sigma_{i}$ when expressed in the coordinates given by the chart $\rho_{i}$. Likewise, we may assume that $R$ in coordinates $x=\left(x_{s s}, x_{u}, x_{u u}\right)$ introduced in Appendix $\mathrm{A}$, acts by $R\left(x_{s s}, x_{u}, x_{u u}\right)=\left(x_{u u}, x_{u}, x_{s s}\right)$. Note that $\Sigma_{i}$ is not near the fixed point $p$. 


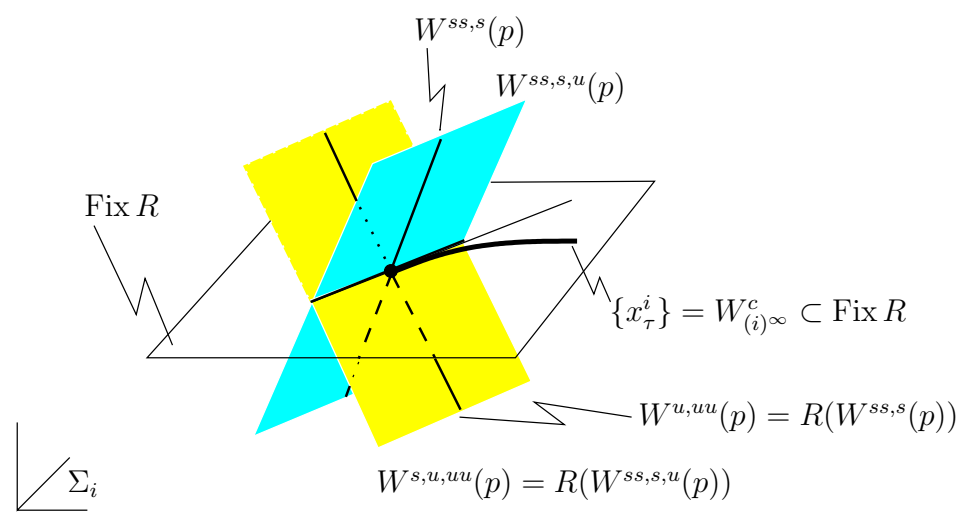

Figure 2. This figure illustrates the geometry of the different invariant manifolds within the cross-section $\Sigma_{i}$ and Fix $R$.

The return map $\Psi$ on $\Sigma_{1} \cup \Sigma_{2}$ is reversible,

$$
\Psi R=R \Psi^{-1} .
$$

Observe that $\Psi$ consists of four maps $\Psi^{i, j}: \Sigma_{i} \rightarrow \Sigma_{j}, i, j=1,2$. Note that (8) implies $\Psi^{n} R=R \Psi^{-n}$. Therefore, $\Psi^{n} R$ is an involution for each $n$ and $\Psi$ is reversible with respect to it:

$$
\Psi\left(\Psi^{n} R\right)=\left(\Psi^{n} R\right) \Psi^{-1}
$$

see also Dev58, Dev76.

We will show that symmetric periodic orbits occur along one-parameter families. For this we define symmetric itineraries which are related to symmetric orbits of the vector field. Define an involution $\mathcal{R}$ on $\mathcal{B}_{2}$, the space of double infinite sequences of two symbols $\{1,2\}$, as follows:

$$
\mathcal{R} \eta(k)=\eta(-k) .
$$

For each orbit $x, \Upsilon(R x)=\mathcal{R} \Upsilon(x)$. As explained in Appendix C,

$$
W_{\mathcal{R} \eta}^{c}=R W_{\eta}^{c} \text {. }
$$

Note that the shift $\sigma$ on $\mathcal{B}_{2}$ is reversible with respect to the involution $\mathcal{R}$. We call an itinerary $\eta$ symmetric if there exists $s \in \mathbb{Z}$ such that

$$
\mathcal{R} \eta=\sigma^{s} \eta
$$

The set of symmetric itineraries comprises the set of those whose $\sigma$-orbit is symmetric. Moreover, if $\eta$ is a symmetric $N$-periodic orbit, then $\mathcal{R} \eta=\sigma^{s} \eta$ for some $0<s \leq N$.

An itinerary $\eta$ is called almost periodic, if each finite block $\eta(s), \ldots, \eta(s+N)$ occurs infinitely often in $\eta$ and there is $M>0$ so that between any two occurrences, there are at most $M$ symbols. Note that $\eta$ is a minimal orbit under the action of the shift operator $\sigma$ MorHed38. It is shown in MorHed38 that periodic and almost periodic sequences provide all periodic and non-periodic minimal sequences. An almost periodic itinerary is called symmetric if its $\sigma$-orbit is accumulated by $\sigma$-orbits of symmetric periodic itineraries. 
Theorem 4.2 below gives some symbolic sequences that occur as itineraries of nonwandering points for $\Psi$. It implies that there are families of symmetric $N$ periodic orbits, but the existence of (families of) symmetric non-periodic orbits cannot be guaranteed. The geometrical reason for that is the (possible) absence of a first integral which enforces invariant level sets.

Theorem 4.2. For each symmetric periodic or almost periodic itinerary $\eta \in \mathcal{B}_{2}$, there is a one-parameter family of orbits $x_{\lambda} \in W_{\eta}^{c}$, with $\Upsilon\left(x_{\lambda}\right)=\eta$. Further, $x_{\lambda}$ is a periodic orbit if $\eta$ is periodic and $x_{\lambda}$ is a minimal orbit if $\eta$ is almost periodic.

Proof. Consider first a periodic symmetric itinerary $\eta$ of period $N$. By (10), there is an $0 \leq s<N$ such that $W_{\mathcal{R} \eta}^{c}=W_{\sigma^{s} \eta}^{c}$. Hence $R W_{\eta}^{c}$ equals $\Psi^{s}\left(W_{\eta}^{c}\right)$. It follows that $\Psi^{k} R\left(W_{\eta}^{c}\right)=W_{\eta}^{c}$ with $k=N-s$. Note that $\gamma_{\eta(0)}$ is a fixed point of $U_{k}=\Psi^{k} R$ on $W_{\eta}^{c}$. Because of this, the involution $U_{k}$ can only leave the curve $W_{\eta}^{c}$ invariant, if it fixes each point on $W_{\eta}^{c}$. Thus $W_{\eta}^{c} \subset$ Fix $U_{k}$. Because $\Psi^{N}$ maps $W_{\eta}^{c}$ into itself and $\Psi^{N}$ is reversible with respect to $U_{k}$, the center manifold $W_{\eta}^{c}$ consists of periodic points: orbits that intersect the fixed space of an involution twice are symmetric periodic orbits. Moreover, these are all periodic points of period $N$, if $N$ is the minimal period of $\eta$.

Consider next a symmetric almost periodic itinerary $\eta$. There is a sequence $\eta_{n}$ of symmetric periodic itineraries so that their $\sigma$-orbits converge to the $\sigma$-orbit of $\eta$. Denote by $X_{n}$ the branches of periodic orbits $x_{n}$ with $\Upsilon\left(x_{n}\right)$ equal to a shift of $\eta_{n}$. Let $y$ be an accumulation point of $X_{n} \cap\left\{x_{u}=r\right\}$ for some $0<r<\delta$. Since almost periodic itineraries are minimal, the point $y$ lies on a minimal orbit. It follows that $W_{\eta}^{c}$ contains a branch of recurrent points with itinerary $\eta$. The orbits of these points are minimal orbits.

We remark that, apart from periodic and almost periodic orbits, homoclinic and heteroclinic connections between periodic and almost periodic orbits, corresponding to itineraries with a periodic or an almost periodic past and future, also occur along one-parameter families. This follows from transversality of center stable and center unstable manifolds.

Theorem 4.3. For $X$ from a residual set of vector fields, non-symmetric periodic orbits are hyperbolic.

Proof. This result follows from the Kupka-Smale theorem for reversible systems in Dev76.

\section{NON-SYMMETRIC HOMOCLINIC ORBITS IN REVERSIBLE SYSTEMS}

Consider a vector field $X$ on a $2 n$-dimensional Riemannian manifold $M$ that is reversible with respect to an involution $R$ :

$$
D R(x) X(x)=-X \circ R(x),
$$

as in Section 4

In this section we assume that $X$ has a non-symmetric homoclinic orbit $\Gamma_{1}$, connecting the symmetric equilibrium $p$. By reversibility, $X$ possesses a second homoclinic orbit $\Gamma_{2}=R\left(\Gamma_{1}\right)$. As before, we assume that the transversality conditions (3) and (4) hold. We also assume (5). And we assume that $\{p\} \cup \Gamma_{1} \cup \Gamma_{2}$ forms a homoclinic bellows. For this it is necessary that $D R(p) e^{u}=-e^{s}$ (and not $\left.D R(p) e^{u}=e^{s}\right)$. See (11) and (2) for the definition of $e^{s}, e^{u}$. 
In contrast to the bellows in Section 4 the homoclinic orbits do not persist under perturbations of $X$. One is led to consider one-parameter families of vector fields that unfold the homoclinic bifurcation. Let $\left\{X_{\mu}\right\}$ be a one-parameter family of vector fields with $X_{0}=X$. Take a cross-section $\Sigma_{1}$ transverse to $\Gamma_{1}$. The crosssection $\Sigma_{2}=R\left(\Sigma_{1}\right)$ is transverse to $\Gamma_{2}$. Let $\Psi_{\mu}$ be the return map for $X_{\mu}$ on $\Sigma_{1} \cup \Sigma_{2}$. Again, as in the case of symmetric homoclinic orbits, $\Psi_{\mu}$ is reversible. The return map $\Psi_{\mu}$ consists of four maps $\Psi_{\mu}^{i, j}: \Sigma_{i} \rightarrow \Sigma_{j}, i, j=1,2$.

Appendix $\mathrm{A}$ contains asymptotic expansions for $\Psi_{\mu}^{i, j}$ in Shil'nikov variables. We may assume that a coordinate system on $\Sigma_{2}$ is obtained from coordinates on $\Sigma_{1}$ by composing with $R$. From Theorem A.6 and the remark at the end of Appendix A, it follows that coordinates $x^{i}=\left(x_{s s}^{i}, x_{u}^{i}, x_{u u}^{i}\right)$ exist on $\Sigma_{i}, i=1,2$, with the following properties (for the precise meaning of the notation, see Notation A.5). The action of $R$ is given by $R\left(x_{s s}^{1}, x_{u}^{1}, x_{u u}^{1}\right)=\left(x_{u u}^{2}, x_{u}^{2}, x_{s s}^{2}\right)$. In the following we will suppress the superscript indicating the cross-section. If $x(k+1)=\Psi(x(k))$ with $x(k+1) \in \Sigma_{i}$ and $x(k) \in \Sigma_{j}$, then

$$
\begin{aligned}
x_{s s}(k+1) & =\mathcal{O}\left(x_{u}(k)^{1+\omega}\right), \\
x_{u}(k+1) & =a_{i, j}(\mu)+\varphi_{i, j}(\mu) x_{u}(k)+\mathcal{O}\left(x_{u}(k)^{1+\omega}\right), \\
x_{u u}(k) & =\mathcal{O}\left(x_{u}(k+1)^{1+\omega}\right),
\end{aligned}
$$

for some $\omega>0$. By reversibility,

$$
\begin{array}{cc}
a_{1,1}=-a_{2,2}, & a_{1,2}=a_{2,1}=0, \\
\varphi_{1,1}=1 / \varphi_{2,2}, & \varphi_{1,2}=\varphi_{2,1}=1 .
\end{array}
$$

Note that $\varphi_{1,2}$ and $\varphi_{2,1}$ cannot be equal to -1 , due to (5). We will assume

$$
\begin{aligned}
D a_{1,1}(0) & >0, \\
\varphi_{1,1}(0) & >1 .
\end{aligned}
$$

These assumptions are generic, since we can change the inequalities by relabeling $\Gamma_{1}$ and $\Gamma_{2}$ and redefining $\mu$.

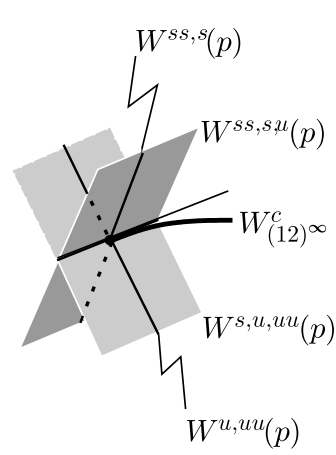

$\Sigma_{1}$

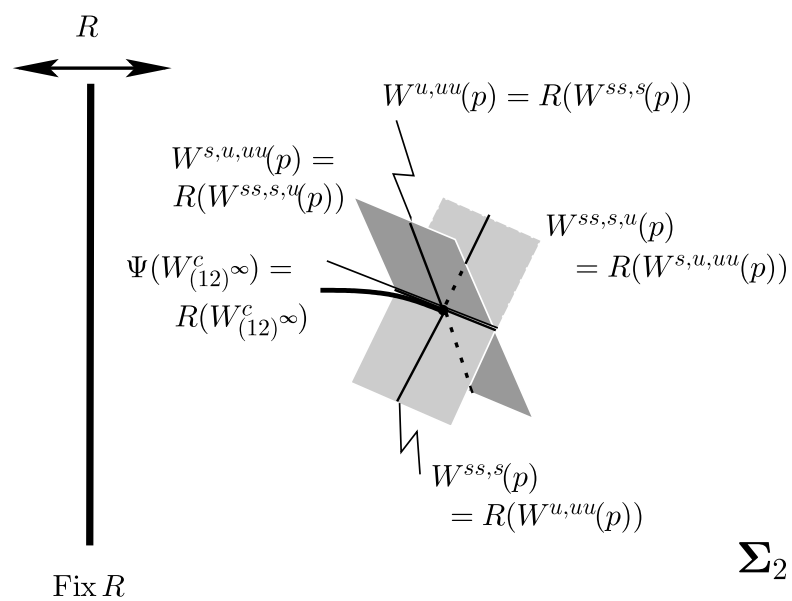

Figure 3. This figure illustrates the geometry, at $\mu=0$, of the different invariant manifolds with respect to the cross-sections $\Sigma_{1}$ and $\Sigma_{2}$. 
Recall that $\mathcal{B}_{2}$ denotes the space of double infinite sequences of two symbols $\{1,2\}$, equipped with the product topology. Define the involution $\mathcal{R}_{1}$ on $\mathcal{B}_{2}$ by

$$
\mathcal{R}_{1} \eta(k)= \begin{cases}1, & \text { if } \eta(\mathrm{k})=2 \\ 2, & \text { if } \eta(\mathrm{k})=1\end{cases}
$$

Further, we consider another involution $\mathcal{R}_{2}$ on $\mathcal{B}_{2}$

$$
\mathcal{R}_{2} \eta(k)=\eta(-k) .
$$

The composition $\mathcal{R}=\mathcal{R}_{1} \circ \mathcal{R}_{2}$ is again an involution and the shift $\sigma$ on $\mathcal{B}_{2}$ is reversible with respect to $\mathcal{R}$. As in the previous section, $\Upsilon(R x)=\mathcal{R} \Upsilon(x)$ and

$$
W_{\mathcal{R} \eta}^{c}=R W_{\eta}^{c} .
$$

Again we call an itinerary $\eta$ symmetric if $\mathcal{R} \eta=\sigma^{s} \eta$ for some $s \in \mathbb{Z}$. Almost periodic itineraries (see the previous section) are called symmetric if they are accumulated by $\sigma$-orbits of symmetric periodic orbits.

For a periodic itinerary $\eta$ of period $N$, let $s(\eta)$ be the difference of the number of 1 's and the number of 2's in $\eta(0), \ldots, \eta(N-1)$. Thus $s(\eta)=0$ if $\eta(0), \ldots, \eta(N-1)$ contains as many 1's as 2's. In particular, $s(\eta)=0$ for symmetric itineraries. But non-symmetric itineraries with $s$ vanishing exist as well, for example $\eta=$ $(11211222)^{\infty}$.

The dynamics of a vector field with bellows consisting of a non-symmetric homoclinic orbit and its symmetric image, as well as of nearby vector fields, is discussed in the following results.

Theorem 5.1. Assume that (13) and (14) hold.

Let $\eta \in \mathcal{B}_{2}$ be a symmetric periodic itinerary of period $N$. For each such $\eta$, there is an interval $I=\left(-\mu^{*}, \mu^{*}\right)$, so that for each $\mu \in I$, there exists a family of $N$-periodic orbits $\left\{x_{\lambda}\right\}$ contained in $W_{\eta}^{c}$, with $\Upsilon\left(x_{\lambda}\right)=\eta$. For $\mu=0$, the family $\left\{x_{\lambda}\right\}$ is bounded by the bellows. For $\mu \neq 0$, it is bounded by a finite collection of symmetric homoclinic orbits.

Let $\eta \in \mathcal{B}_{2}$ be a non-symmetric periodic itinerary of period $N$, such that $s(\eta) \neq 0$. For $\mu \leq 0$, there is no periodic orbit with $\eta$ as its itinerary. For each such $\eta$, there is an interval $I=\left(0, \mu^{*}\right)$, so that for each $\mu \in I$, there is a unique hyperbolic $N$ periodic orbit $x$ with $\Upsilon(x)=\eta$. The hyperbolic periodic orbits with itinerary $\eta$ and $\mathcal{R} \eta$ have different index. There exists a heteroclinic cycle connecting these periodic orbits.

Proof. For $\mu=0$, the statements concerning symmetric periodic orbits are verbatim the same as Theorem 4.2 only the involution used in the definition of symmetric itinerary has changed. The proof can be copied from Theorem 4.2 as well. Note that the proof also predicts the existence of symmetric almost periodic orbits.

Next, let $\mu \neq 0$ and consider a symmetric periodic itinerary $\eta$ of period $N$. Consider the return map $\Psi_{0}^{N}$ restricted to $W_{\eta}^{c}$. Then $\left.\Psi_{0}^{N}\right|_{W_{\eta}^{c}}$ is the identity map, whenever it is defined. These points yield periodic orbits with itinerary $\eta$. For small values of $\mu, \Psi_{0}^{N} \mid W_{\eta}^{c}$ is clearly well defined for sufficiently large values of $x_{u}$, as it is for $\mu=0$. The point with smallest coordinate for which $\left.\Psi_{0}^{N}\right|_{W_{\eta}^{c}}$ is defined, is a point which is in the stable manifold of $p$, and lies on a homoclinic orbit since it is accumulated by periodic orbits. The family of periodic orbits with itinerary $\eta$ is therefore bounded by a finite union of homoclinic orbits. 
To prove the other statements, we will iterate points on the Cantor bouquet of center manifolds. For our calculations we will parametrize the center manifolds by the coordinate $x_{u}$. Write $x_{u} \mapsto f_{\eta}\left(x_{u}\right)$ for the return map $\Psi_{\mu}: W_{\eta}^{c} \rightarrow W_{\sigma \eta}^{c}$. Note that $f_{\eta}$ is continuously differentiable, also in the parameter $\mu$. By Theorem 2.2 , $W_{\eta}^{c}$ is the graph of a map $w_{\eta}^{c}: E^{u} \rightarrow E^{s s} \times E^{u u}$. Putting the equations for the center manifolds $W_{\eta}^{c}$ into $\Psi_{\mu}$, applying Theorem A.6 one obtains the following asymptotics for $f_{\eta}$ :

$$
f_{\eta}(x)=a_{\eta(0), \eta(1)}(\mu)+\varphi_{\eta(0), \eta(1)}(\mu) x+r_{\eta}(x),
$$

where $\left|r_{\eta}(x)\right| \leq C x^{1+\omega}$ for some $C>0, \omega>0$, uniformly in $\mu$ and $\eta$. By the remark following the proof of Theorem 2.2 in Appendix $\mathbf{C} .\left|D w_{\eta}^{c}\right|$ is bounded uniformly in $\mu$ and $\eta$. Applying Theorem A.6 and the chain rule gives that one also has a bound $\left|D r_{\eta}(x)\right| \leq C x^{\omega}$, for some $C>0, \omega>0$, uniformly in $\mu$ and $\eta$.

To establish the claims on the appearance of non-symmetric periodic orbits, consider, for a non-symmetric periodic itinerary $\eta$ of period $N$, the return map $\Psi_{0}^{N}$ restricted to $W_{\eta}^{c}$. This return map is given by $x_{u} \mapsto F_{\mu}\left(x_{u}\right)=f_{\sigma^{N-1} \eta} \circ \ldots \circ f_{\eta}\left(x_{u}\right)$. Observe that $F_{\mu}$ satisfies

$$
\begin{aligned}
F_{0}(0) & =0, \\
D F_{0}(0) & =\varphi_{1,1}(0)^{s(\eta)} .
\end{aligned}
$$

By assumption, $s(\eta) \neq 0$. We may suppose that $s(\eta)>0$, otherwise consider the itinerary $\mathcal{R} \eta$ instead of $\eta$. Then $F_{\mu}$ is an increasing function with $D F_{\mu}$ close to $\varphi_{1,1}(0)^{s(\eta)}$, which is bigger than 1 , for $\mu$ small. Moreover, $F_{\mu}(0)<0$ if $\mu<0$ and $F_{\mu}(0)>0$ if $\mu>0$. It follows that a hyperbolic periodic orbit $\mathfrak{p}$ with itinerary $\eta$, does not exist if $\mu \leq 0$.

A rescaling makes the calculations to find a hyperbolic periodic orbit $\mathfrak{p}$ with itinerary $\eta$ for $\mu>0$ more illuminating. For $\mu>0$, define a coordinate $\bar{x}_{u}$ by $a_{1,1} \bar{x}_{u}=x_{u}$. In this coordinate, since $a_{1,1}(\mu)=\mathcal{O}(\mu)$, one can write

$$
f_{\eta}\left(\bar{x}_{u}\right)=\frac{a_{\eta(0), \eta(1)}(\mu)}{a_{1,1}}+\varphi_{\eta(0), \eta(1)}(\mu) \bar{x}_{u}+\mathcal{O}\left(\mu^{\omega}\right),
$$

as $\mu \rightarrow 0$. In the limit $\mu \rightarrow 0, f_{\eta}$ depends only on $\eta(0), \eta(1)$ and thus four interval maps occur, namely

$$
\begin{array}{rlrl}
\bar{x}_{u} & \mapsto 1+\varphi_{1,1}(0) \bar{x}_{u}, & & \text { if } \eta(0)=\eta(1)=1, \\
\bar{x}_{u} \mapsto \frac{\left(\bar{x}_{u}-1\right)}{\varphi_{1,1}(0)}, & & \text { if } \eta(0)=\eta(1)=2,
\end{array}
$$

and the identity map otherwise. Note that the two non-trivial maps are linear, and one is the inverse of the other. In particular, they have a common fixed point at $x=1 /\left(1-\varphi_{1,1}(0)\right)$. This makes clear that, for $\mu$ small and positive, $F_{\mu}$ has a unique repelling fixed point $\rho(\mu)$ with $\lim _{\mu \downarrow 0} \rho(\mu) / a_{1,1}(\mu)=1 /\left(1-\varphi_{1,1}(0)\right)$. An open neighborhood of $\rho(\mu)$ is contained in the domain of definition of $F_{\mu}$, for $\mu$ small enough.

Observe that the periodic orbit $\mathfrak{p}$ has $n$-dimensional unstable manifold and $(n-$ 1)-dimensional stable manifold. Its symmetric image $R \mathfrak{p}$ is a hyperbolic periodic orbit with $(n-1)$-dimensional unstable manifold and $n$-dimensional stable manifold.

Finally, we prove the existence of a heteroclinic cycle connecting $\mathfrak{p}$ and $R \mathfrak{p}$. Write $A=\eta(0) \cdots \eta(N-1)$ and $B=R \eta(0) \cdots R \eta(N-1)$. Let $\xi=\cdots A A A B B B \cdots$. Note that $\xi$ is symmetric. For $\mu$ small and positive, the local unstable manifold of 
$\mathfrak{p}$ intersects $W_{\sigma^{-s} \xi}^{c}$ with $s$ large and positive, in an open interval containing $\rho(\mu)$. Indeed, the center manifolds $W_{\sigma^{-s} \xi}^{c}$ are contained in the center unstable manifold $W_{\eta}^{c, u u}$ corresponding to the itinerary $\eta$ (see the construction of center manifolds in Appendix (C). An iterate of $\Psi$ maps the local unstable manifold of $\mathfrak{p}$ intersected with $W_{\xi}^{c}$ to the local stable manifold of $R \mathfrak{p}$ intersected with $W_{\sigma \xi}^{c}$, by reversibility this yields heteroclinic connections. Similarly, one obtains a heteroclinic connection from $R \mathfrak{p}$ to $\mathfrak{p}$, with itinerary $\chi=\cdots B B B A A A \cdots$. Here a unique connection is obtained since $\mathfrak{p}$ has a one-dimensional stable manifold contained in the center stable manifold $W_{\eta}^{s s, c}$. The stable manifold of $\mathfrak{p}$ intersects the center manifolds $W_{\sigma^{s} \chi}^{c}, s$ large and positive, transversally within $W_{\eta}^{s s, c}$.

The interesting feature of the heteroclinic cycles connecting a hyperbolic periodic orbit and its symmetric image, is that it connects two orbits with different index. Periodic orbits not discussed in the above theorem, the non-symmetric ones with the value of $s$ vanishing, are (if they exist) hyperbolic for generic vector fields by Theorem 4.3 .

The above result shows that symmetric periodic orbits form sheets, bounded by a collection of homoclinic orbits. This raises the question of which homoclinic orbits exist. If $\mu=0, \Gamma_{1}$ and $\Gamma_{2}$ are the only homoclinic orbits. Which homoclinic orbits one finds for $\mu \neq 0$, is answered in the following theorem.

Homoclinic orbits have finite itineraries. To a finite itinerary $\zeta$, there corresponds a periodic itinerary $\zeta^{\infty}$ in which $\zeta$ is repeated. We call a finite itinerary $\zeta$ symmetric if $\zeta^{\infty}$ is symmetric. Let $\zeta$ be a finite itinerary of length $N$. We call $\zeta 1$-minimal if for all $0 \leq s<N$, the number of 1's is strictly larger than the number of 2's in $\zeta(i)$, $0 \leq i \leq s$. We say that $\zeta$ is 2 -minimal if this condition holds with the role of 1 's and 2's interchanged. For example, $\zeta=12$ is a 1 -minimal itinerary, while $\zeta=21$ is a 2-minimal itinerary.

Theorem 5.2. Let $\zeta$ be a 1-minimal symmetric itinerary of length $N$. Then there is an interval $I=\left(0, \mu^{*}\right)$, so that for $\mu \in I$, there is a homoclinic orbit with itinerary $\zeta$.

Let $\zeta$ be a 2-minimal symmetric periodic itinerary of period $N$. Then there is an interval $I=\left(\mu^{*}, 0\right)$, so that for $\mu \in I$, there is a homoclinic orbit with itinerary $\zeta$.

Proof. Let $\zeta$ be a 1-minimal symmetric itinerary of length $N$. Write $\eta=\zeta^{\infty}$. Iterate the maps $f_{\sigma^{s} \eta}, 0<s \leq N-1$, for $\mu$ small and positive. In the limit $\mu \rightarrow 0$, this reduces to iterating the maps (15) and (16). Being 1-minimal implies that $f_{\sigma^{s} \eta} \circ \ldots \circ f_{\eta}(0)$ is bounded away from 0 for any $0<s<N-1$. The sheet of periodic orbits with itinerary $\eta$ is therefore bounded by a single homoclinic orbit, with itinerary $\zeta$.

Take $\mu>0$. Suppose that $\zeta$ is finite symmetric itinerary of length $N$. Assume that $\zeta$ is not 1-minimal, but starts with a symmetric 1-minimal piece $\zeta(0) \cdots$ $\zeta(M-1)$ with $M<N$. We claim that there is no homoclinic orbit with itinerary $\zeta$. The itinerary $\zeta(0) \cdots \zeta(M-1)$ gives rise to a homoclinic orbit. Consider the component of $\Psi_{\mu}^{M}\left(W^{u, u u}(p) \cap \Sigma_{1}\right)$ with itinerary $\zeta$. Then $\Psi_{\mu}^{M}\left(W^{u, u u}(p) \cap \Sigma_{1}\right)=$ $W^{u, u u}(p) \cap \Sigma_{1}$, so that there cannot be a homoclinic orbit with itinerary $\zeta$.

It does not appear possible to list all homoclinic orbits that occur for $\mu$ small. For instance, the sheet of periodic orbits with itinerary $(1121122211122122)^{\infty}$ might 
be bounded by a single symmetric homoclinic orbit, or by two non-symmetric homoclinic orbits with itineraries 11211222 and 11122122, depending on details of the vector fields $X_{\mu}$. Note that both itineraries have the same number of 1's and 2's.

The coexistence of several homoclinic orbits for $\mu \neq 0$, described in Theorem 5.2 , shows that symmetric homoclinic bellows are present for $\mu \neq 0$. The results in Section 4 are applicable to these bellows.

\section{Appendix A. Coordinate systems near homoclinic orbits}

This section develops a normal form for a return map, in so-called Shil'nikov variables, near a homoclinic orbit to an equilibrium with real weakest eigenvalues. We do not restrict to reversible systems. The effort done in this section to obtain a simple normal form, makes constructions in other sections more amenable. This is, in particular, true for the construction of center manifolds in Appendix C and subsequently the analysis of the dynamics on the center manifolds (for instance, for the explicit calculations in Section (5).

Let $X$ be a smooth vector field on a finite dimensional Riemannian manifold $M$. Assume that $X$ possesses a hyperbolic equilibrium $p$, and that $D X(p)$ has eigenvalues $\left\{\lambda_{i}^{s s}\right\}_{1 \leq i \leq q_{s s}}, \lambda^{s}, \lambda^{u},\left\{\lambda_{i}^{u u}\right\}_{1 \leq i \leq q_{u u}}$ with

$$
\operatorname{Re}\left(\lambda_{i}^{s s}\right)<\lambda^{s}<0<\lambda^{u}<\operatorname{Re}\left(\lambda_{i}^{u u}\right) .
$$

Let $T_{p} M=E^{s s} \oplus E^{s} \oplus E^{u} \oplus E^{u u}$ be the resulting $D X(p)$ invariant splitting of $T_{p} M$. Assume that $X$ has a homoclinic orbit $\Gamma$, so that

$$
\begin{gathered}
\lim _{t \rightarrow \infty} \dot{\Gamma}(t) /\|\dot{\Gamma}(t)\| \in E^{s}, \\
\lim _{t \rightarrow-\infty} \dot{\Gamma}(t) /\|\dot{\Gamma}(t)\| \in E^{u} .
\end{gathered}
$$

Take coordinates $\left(x_{s s}, x_{s}, x_{u}, x_{u u}\right)$ near $p$ so that $\left\{x_{s}, x_{u}, x_{u u}=0\right\}$ is tangent to $E^{s s}$ and so on. Following [OvsShi87, we set up suitable local coordinates near $p$.

Lemma A.1. There is a smooth local coordinate system $x=\left(x_{s s}, x_{s}, x_{u}, x_{u u}\right)$ near $p$ in which the vector field $X$ is given by the set of differential equations

$$
\begin{aligned}
\dot{x}_{s s} & =A^{s s} x_{s s}+f^{s s}(x), \\
\dot{x}_{s} & =\lambda^{s} x_{s}+f^{s}(x), \\
\dot{x}_{u} & =\lambda^{u} x_{u}+f^{u}(x), \\
\dot{x}_{u u} & =A^{u u} x_{u u}+f^{u u}(x),
\end{aligned}
$$

where the quadratic and higher order terms $f^{s s}(x), \ldots, f^{u u}(x)$ satisfy

$$
\begin{aligned}
f^{s s}(x) & =\mathcal{O}\left(\left\|x_{s s}\right\|\|x\|\right)+\mathcal{O}\left(x_{s}^{2}\right), \\
f^{s}(x) & =\mathcal{O}\left(\left\|x_{s s, s}\right\|\|x\|\right),
\end{aligned}
$$

as $\left\|x_{s s, s}\right\| \rightarrow 0$ and

$$
\begin{aligned}
f^{u}(x) & =\mathcal{O}\left(\left\|x_{u, u u}\right\|\|x\|\right), \\
f^{u u}(x) & =\mathcal{O}\left(\left\|x_{u u}\right\|\|x\|\right)+\mathcal{O}\left(x_{u}^{2}\right),
\end{aligned}
$$

as $\left\|x_{u, u u}\right\| \rightarrow 0$.

Proof. Note that near $p$ there exist a (locally invariant) stable manifold $W^{s s, s}(p)$ and an unstable manifold $W^{u, u u}(p)$. The stable and unstable manifold are both smooth manifolds. Further, there is a codimension- $q_{s s}$ center unstable manifold 
$W^{s, u, u u}(p)$ with tangent space $E^{s} \oplus E^{u} \oplus E^{u u}$ at $p$, and a codimension- $q_{u u}$ center stable manifold $W^{s s, s, u}(p)$ with tangent space $E^{s s} \oplus E^{s} \oplus E^{u}$ at $p$. The tangent bundles $T_{W^{s s, s}(p)} W^{s s, s, u}(p)$ and $T_{W^{u, u u}(p)} W^{s, u, u u}(p)$ are smooth bundles; see e.g. Hom96. We may thus take smooth coordinates $x=\left(x_{s s}, x_{s}, x_{u}, x_{u u}\right)$ near $p$, so that

$$
\begin{aligned}
& W^{s s, s}(p) \quad=\quad\left\{x_{u}, x_{u u}=0\right\}, \\
& W^{u, u u}(p)=\left\{x_{s s}, x_{s}=0\right\}, \\
& W^{s s, s, u}(p) \bar{\Pi}_{W^{s s, s}(p)}\left\{x_{u u}=0\right\}, \\
& W^{s, u, u u}(p) \bar{\Pi}_{W^{u, u u}(p)}\left\{x_{s s}=0\right\} .
\end{aligned}
$$

The notation $W \bar{\cap}_{q} V$ means that $W$ is tangent to $V$ at $q$. From (18) it follows that $f^{s s}$ and $f^{s}$ are $\mathcal{O}\left(\left\|x_{s s, s}\right\|\right)$. Similarly, from (17) one gets that $f^{u}$ and $f^{u u}$ are $\mathcal{O}\left(\left\|x_{u, u u}\right\|\right)$. Write $X=\left(X^{s s}, X^{s}, X^{u}, X^{u u}\right)$. From (20) and (19) one sees that

$$
\begin{aligned}
\left.D X^{s s, s}\right|_{x_{s s}, x_{s}=0} & =\left(\begin{array}{cc}
A^{s s}+\mathcal{O}\left(\left\|x_{u, u u}\right\|\right) & 0 \\
\mathcal{O}\left(\left\|x_{u, u u}\right\|\right) & \lambda^{s}+\mathcal{O}\left(\left\|x_{u, u u}\right\|\right)
\end{array}\right), \\
\left.D X^{u, u u}\right|_{x_{u}, x_{u u}=0} & =\left(\begin{array}{cc}
\lambda^{u}+\mathcal{O}\left(\left\|x_{s s, s}\right\|\right) & \mathcal{O}\left(\left\|x_{s s, s}\right\|\right) \\
0 & A^{u u}+\mathcal{O}\left(\left\|x_{s s, s}\right\|\right)
\end{array}\right),
\end{aligned}
$$

respectively. The lemma follows.

Let $\Sigma^{i n} \subset\left\{x_{s}=\delta\right\}, \Sigma^{\text {out }} \subset\left\{x_{u}=\delta\right\}$ be cross-sections close to $p$. By a rescaling we may assume $\delta=1$. Write $x^{i n}=\left(x_{s s}^{i n}, x_{u}^{i n}, x_{u u}^{i n}\right)$ for the coordinate system on $\Sigma^{\text {in }}$ inherited from the coordinates near $p$. Write similarly $\left(x_{s s}^{\text {out }}, x_{s}^{\text {out }}, x_{u u}^{\text {out }}\right)$ for coordinates on $\Sigma^{o u t}$. The following theorem discusses the first hit map $\Psi: \Sigma^{i n} \rightarrow$ $\Sigma^{\text {out }}$ in Shil'nikov variables [Shi65, Shi67]; see also [Den89, Den89b] and Hom00] for the specific formulation used here.

Proposition A.2. Let $\Psi: \Sigma^{i n} \rightarrow \Sigma^{\text {out }}$ be the first hit map given by the flow of $X$. Write $\beta=-\lambda^{s} / \lambda^{u}$. In coordinates near $p$ as in Lemma A.1, $\Psi\left(x_{s s}^{i n}, x_{u}^{i n}, x_{u u}^{i n}\right)=$ $\left(x_{s s}^{\text {out }}, x_{s}^{\text {out }}, x_{u u}^{\text {out }}\right)$ satisfies the following. There exists a map $S$ so that we can write

$$
\left(x_{s s}^{\text {out }}, x_{s}^{\text {out }}, x_{u u}^{\text {in }}\right)=S\left(x_{s s}^{\text {in }}, x_{u}^{\text {in }}, x_{u u}^{\text {out }}\right) .
$$

There exists $\omega>0$, so that as $x_{u} \rightarrow 0, S=\left(S^{s s}, S^{s}, S^{u u}\right)$ satisfies

$$
\begin{aligned}
\left|D_{x_{u}^{\text {in }}}^{k} D_{x_{s s}^{\text {out }}, x_{u u}^{\text {in }}}^{l} D^{m} S^{s s}\left(x_{s s}^{\text {in }}, x_{u}^{\text {in }}, x_{u u}^{\text {out }}\right)\right| & \leq C_{k+l+m}\left|x_{u}^{\text {in }}\right|^{\beta+\omega-k}, \\
\left|D_{x_{u}^{\text {in }}}^{k} D_{x_{s s}^{\text {out }}, x_{u u}^{\text {in }}}^{l} D^{m}\left(S^{s}\left(x_{s s}^{\text {in }}, x_{u}^{\text {in }}, x_{u u}^{\text {out }}\right)-\left(x_{u}^{\text {in }}\right)^{\beta} \varphi\left(x_{s s}^{\text {in }}, x_{u u}^{\text {out }}\right)\right)\right| & \leq C_{k+l+m}\left|x_{u}^{\text {in }}\right|^{\beta+\omega-k}, \\
\left|D_{x_{u}^{\text {in }}}^{k} D_{x_{s s}^{\text {out }}, x_{u u}^{\text {in }}}^{l} D^{m} S^{u u}\left(x_{s s}^{\text {in }}, x_{u}^{\text {in }}, x_{u u}^{\text {out }}\right)\right| & \leq C_{k+l+m}\left|x_{u}^{\text {in }}\right|^{1+\omega-k},
\end{aligned}
$$

for positive constants $C_{k+l+m}$. Here $\varphi$ is a smooth nonvanishing map.

Proof. The proof that follows is essentially contained in Den89, Den96.

On a compact neighborhood of the origin that includes $\Sigma^{i n}$ and $\Sigma^{\text {out }}$, there is $K>0$ so that

$$
\begin{aligned}
\left\|f^{s s}(x)\right\| & \leq K \delta\left(\left\|x_{s s}\right\|\|x\|+x_{s}^{2}\right), \\
\left|f^{s}(x)\right| & \leq K \delta\left(\left\|x_{s s, s}\right\|\|x\|\right), \\
\left|f^{u}(x)\right| & \leq K \delta\left(\left\|x_{u, u u}\right\|\|x\|\right), \\
\left\|f^{u u}(x)\right\| & \leq K \delta\left(x_{u}^{2}+\left\|x_{u u}\right\|\|x\|\right) .
\end{aligned}
$$


By the variation of constants formula, an orbit $x(t)$ with $x_{s s, s}(0)=x_{s s, s}^{0}$ and $x_{u, u u}(\tau)=x_{u u, u}^{\tau}$, satisfies

$$
\begin{aligned}
x_{s s}(t) & =e^{A^{s s} t} x_{s s}^{0}+\int_{0}^{t} e^{A^{s s}(t-v)} f^{s s}(x(v)) d v, \\
x_{s}(t) & =e^{\lambda^{s} t}+\int_{0}^{t} e^{\lambda^{s}(t-v)} f^{s}(x(v)) d v, \\
x_{u}(t) & =e^{\lambda^{u}(t-\tau)}-\int_{t}^{\tau} e^{\lambda^{u}(t-v)} f^{u}(x(v)) d v, \\
x_{u u}(t) & =e^{A^{u u}(t-\tau)} x_{u u}^{\tau}-\int_{t}^{\tau} e^{A^{u u}(t-v)} f^{u u}(x(v)) d v .
\end{aligned}
$$

Let $\omega$ be a small positive number and consider the space $E$ of functions $\mathbf{x}=$ $\{x(t), 0 \leq t \leq \tau\}$ for which

$$
\begin{aligned}
\left\|x_{s s}(t)\right\| e^{-\left(\lambda^{s}-\omega\right) t} & <\infty, \\
\left|x_{s}(t)\right| e^{-\lambda^{s} t} & <\infty, \\
\left|x_{u}(t)\right| e^{-\lambda^{u}(t-\tau)} & <\infty, \\
\left\|x_{u u}(t)\right\| e^{-\left(\lambda^{u}+\omega\right)(t-\tau)} & <\infty .
\end{aligned}
$$

Equip $E$ with the norm

$$
\begin{aligned}
\|\mathbf{x}\|=\sup _{0 \leq t \leq \tau}\left\{\left\|x_{s s}(t)\right\| e^{-\left(\lambda^{s}-\omega\right) t},\left|x_{s}(t)\right| e^{-\lambda^{s} t},\left|x_{u}(t-\tau)\right| e^{-\lambda^{u}(t-\tau)},\right. \\
\left.\left\|x_{u u}(t-\tau)\right\| e^{-\left(\lambda^{u}+\omega\right)(t-\tau)}\right\} .
\end{aligned}
$$

The right-hand side of (21)-(24) defines a map $\mathcal{G}$ on $E$. Write $B_{r}=\{\mathbf{x} \in E ;\|\mathbf{x}\| \leq$ $r\}$ for the ball with radius $r$ in $E$. We claim that $\mathcal{G}$ is a contraction on some ball in $E$ : there exist $r>0$ and $0<\lambda<1$, so that

- $\mathcal{G}\left(B_{r}\right) \subset B_{r}$.

- $\left\|\mathcal{G}\left(\mathbf{x}_{1}\right)-\mathcal{G}\left(\mathbf{x}_{2}\right)\right\| \leq \lambda\left\|\mathbf{x}_{1}-\mathbf{x}_{2}\right\|$, for $\mathbf{x}_{1}, \mathbf{x}_{2} \in B_{r}$.

To check the first item, consider the strong stable coordinate $x_{s s}$ of $\mathbf{x} \in B_{r}$. Take $\omega$ small enough so that $\lambda^{s s}<\lambda^{s}-2 \omega$. We may assume that $\left\|e^{s^{s s}} t\right\| \leq e^{\left(\lambda^{s}-2 \omega\right) t}$. In the following, $C$ will denote a positive constant which can change from line to line, but does not depend on $t$. Now

$$
\begin{aligned}
& \left\|\mathcal{G}_{s s}(\mathbf{x})(t)\right\| \\
& \begin{aligned}
\leq e^{\left(\lambda^{s}-2 \omega\right) t}\left\|x_{s s}^{0}\right\|+\int_{0}^{t} e^{\left(\lambda^{s}-2 \omega\right)(t-v)}\left\|f^{s s}(\mathbf{x}(v))\right\| d v \\
\leq e^{\left(\lambda^{s}-2 \omega\right) t}\left\|x_{s s}^{0}\right\|+\int_{0}^{t} e^{\left(\lambda^{s}-2 \omega\right)(t-v)} C \delta\left(\left\|x_{s s}(v)\right\|\left\|x_{s s, s}(v)\right\|\right. \\
\left.+\left\|x_{s s}(v)\right\|\left\|x_{u, u u}(v)\right\|+\left\|x_{s}(v)\right\|^{2}\right) d v \\
\leq e^{\left(\lambda^{s}-2 \omega\right) t}\left\|x_{s s}^{0}\right\|+\int_{0}^{t} e^{\left(\lambda^{s}-2 \omega\right)(t-v)} C \delta\left(e^{\left(\lambda^{s}-\omega\right) v} e^{\lambda^{s} v}\right. \\
\left.+e^{\left(\lambda^{s}-\omega\right) v} e^{\lambda^{u}(v-\tau)} e^{2 \lambda^{s} v}\right) d v \\
\leq e^{\left(\lambda^{s}-\omega\right) t}\left[C e^{-\omega t}+C \delta\left(e^{-\omega t}+e^{\lambda^{u}(t-\tau)}\right)\right] .
\end{aligned}
\end{aligned}
$$


Corresponding estimates for the other coordinates, $x_{s}(t), x_{u}(t), x_{u u}(t)$ are obtained similarly. The first item holds by taking $r$ large enough. To prove the second item, take $\mathbf{x}_{1}, \mathbf{x}_{2} \in B_{r}$ with $x_{1, s s}(0)=x_{2, s s}(0)$ and $x_{1, u u}(\tau)=x_{2, u u}(\tau)$. Restricting again to the strong stable coordinates,

$$
\begin{aligned}
& \left\|\mathcal{G}_{s s}\left(\mathbf{x}_{1}(t)\right)-\mathcal{G}_{s s}\left(\mathbf{x}_{2}(t)\right)\right\| \\
& \leq \int_{0}^{t} e^{\left(\lambda^{s}-2 \omega\right)(t-v)}\left\|f^{s s}\left(\mathbf{x}_{\mathbf{1}}(v)\right)-f^{s s}\left(\mathbf{x}_{2}(v)\right)\right\| d v \\
& \leq \int_{0}^{t} e^{\left(\lambda^{s}-2 \omega\right)(t-v)} C \delta\left(\left(e^{\lambda^{s} v}+e^{\lambda^{u}(v-\tau)}\right)\left\|x_{1, s s}(v)-x_{2, s s}(v)\right\|\right. \\
& \left.\quad+e^{\lambda^{s} v}\left\|x_{1, s}(v)-x_{2, s}(v)\right\|\right) d v \\
& \leq C \delta e^{\left(\lambda^{s}-\omega\right) t} \sup _{v} e^{\left(-\lambda^{s}+\omega\right) v}\left\|x_{1, s s}(v)-x_{2, s s}(v)\right\| \\
& \quad+C \delta e^{\left(\lambda^{s}-\omega\right) t} \sup _{v} e^{-\lambda^{s} v}\left\|x_{1, s}(v)-x_{2, s}(v)\right\| \\
& \leq C \delta e^{\left(\lambda^{s}-\omega\right) t}\left\|\mathbf{x}_{1}-\mathbf{x}_{2}\right\| .
\end{aligned}
$$

Thus, given $\tau$ large and given $x_{s s}^{0}, x_{u u}^{\tau}$, there is a unique solution to (21)-(24), i.e., a unique orbit $x(t)$ with $x_{s s}(0)=x_{s s}^{0}, x_{u u}(\tau)=x_{u u}^{\tau}$, that needs time $\tau$ to move from $\left\{x_{s}^{\text {in }}=1\right\}$ to $\left\{x_{u}^{\text {out }}=1\right\}$. Moreover, since $\|\mathbf{x}\|$ is bounded, for some $C>0$,

$$
\begin{aligned}
\left\|x_{s s}(t)\right\| & \leq C e^{\left(\lambda^{s}-\omega\right) t}, \\
\left|x_{s}(t)\right| & \leq C e^{\lambda^{s} t}, \\
\left|x_{u}(t)\right| & \leq C e^{\lambda^{u}(t-\tau)}, \\
\left\|x_{u u}(t)\right\| & \leq C e^{\left(\lambda^{u}+\omega\right)(t-\tau)} .
\end{aligned}
$$

Combining the above reasoning with [Den89] (to obtain the leading terms in the exponential expansions of $x_{s}^{\text {out }}$ and $x_{u}^{\text {in }}$ ) and writing $r=e^{-\lambda^{u} \tau}$, it follows that

$$
\begin{aligned}
x_{s s}^{\text {out }} & =r^{-\lambda^{s} / \lambda^{u}} T^{s s}\left(r, x_{s s}^{\text {in }}, x_{u u}^{\text {out }}\right), \\
x_{s}^{\text {out }} & =r^{-\lambda^{s} / \lambda^{u}} \phi^{s}\left(x_{s s}^{\text {in }}, x_{u u}^{\text {out }}\right)+r^{-\lambda^{s} / \lambda^{u}} T^{s}\left(r, x_{s s}^{\text {in }}, x_{u u}^{\text {out }}\right), \\
x_{u}^{\text {in }} & =r \phi^{u}\left(x_{s s}^{\text {in }}, x_{u u}^{\text {out }}\right)+r T^{u}\left(r, x_{s s}^{\text {in }}, x_{u u}^{\text {out }}\right), \\
x_{u u}^{\text {in }} & =r T^{u u}\left(r, x_{s s}^{\text {in }}, x_{u u}^{\text {out }}\right) .
\end{aligned}
$$

Here $\phi^{s}, \phi^{u}$ are smooth functions and $T^{s s}, T^{s}, T^{u}, T^{u u}$ are smooth for $r>0$, with

$$
\left\|D_{x_{s s}^{\text {in }}, x_{u u}^{\text {out }}}^{k}\left(\frac{\partial}{\partial r}\right)^{l} T^{i}\left(r, x_{s s}^{\text {in }}, x_{u u}^{\text {out }}\right)\right\| \leq C_{k+l} r^{\omega / \lambda^{u}-l},
$$

$i=s s, s, u, u u$.

Since $\phi^{u}(0,0) \neq 0$, the function $r$ can be solved from (27) as a function of $x_{s s}^{\text {in }}, x_{u}^{\text {in }}, x_{u u}^{\text {out }}$; see also KisKokOka93b for a calculation in three dimensions. This function is smooth for $x_{u}^{i n}>0$. From (27) and (29) (for $i=u$ ), one estimates that

$$
\left|D_{x_{u}^{i n}}^{k} D_{x_{s s}^{i n}, x_{u u}^{\text {out }}}^{l}\left(r\left(x_{s s}^{\text {in }}, x_{u}^{\text {in }}, x_{u u}^{\text {out }}\right)-\frac{x_{u}^{\text {in }}}{\phi^{u}\left(x_{s s}^{\text {in }}, x_{u u}^{\text {out }}\right)}\right)\right| \leq C_{k+l+m}\left|x_{u}^{\text {in }}\right|^{1+\omega-k} .
$$

Putting this in the remaining formulas (25), (26), (28) proves the proposition. 
Take a cross-section $\Sigma$ transverse to $\Gamma$. Extend the coordinate system $\left(x_{s s}, x_{s}\right.$, $\left.x_{u}, x_{u u}\right)$ near $p$ to a coordinate system near $\{p\} \cup \Gamma$. Suppose that

$$
\begin{array}{ccc}
W^{s s, s, u}(p) & \pitchfork_{\Gamma} & W^{u, u u}(p), \\
W^{s, u, u u}(p) & \pitchfork_{\Gamma} & W^{s s, s}(p) .
\end{array}
$$

The following result can be seen as giving a normal form for a return map on $\Sigma$, in Shil'nikov variables. In its statement, one encounters functions that contain fractional powers of a real positive variable $x$. These functions can be differentiated, losing a power in $x$ with each derivative with respect to $x$. We will use the following notation for this smoothness property.

Notation A.3. Let $(x, y) \mapsto f(x, y)$ with $x \in \mathbb{R}$ be a function that depends smoothly on $y$ and on $x$ for $x>0$. When writing $f(x, y)=\mathcal{O}\left(x^{\alpha}\right)$, we will assume that $\left\|D_{y}^{k} D_{x}^{l} f(x, y)\right\| \leq C_{k+l} x^{\alpha-l}$ for positive constants $C_{k+l}$.

Theorem A.4. Let $\Psi: \Sigma \rightarrow \Sigma$ be the first return map given by the flow of $X$, and let points $x(j), x(j+1)$ in $\Sigma$ be such that $x(j+1)=\Psi(x(j))$. If (30), (31) holds, then a coordinate system $x=\left(x_{s s}, x_{u}, x_{u u}\right)$ on $\Sigma$ can be chosen so that

$$
\begin{array}{cll}
W^{s s, s}(p) \cap \Sigma= & \left\{x_{u, u u}=0\right\}, \\
W^{u, u u}(p) \cap \Sigma= & \left\{x_{s s, u}=0\right\}, \\
W^{s s, s, u}(p) \cap \Sigma \bar{\Pi}_{W^{s s, s}(p) \cap \Sigma} & \left\{x_{u u}=0\right\}, \\
W^{s, u, u u}(p) \cap \Sigma \bar{\cap}_{W^{u, u u}(p) \cap \Sigma} & \left\{x_{s s}=0\right\} .
\end{array}
$$

In these coordinates, $x(j+1)$ and $\Psi(x(j))$ are related by

$$
\left(x_{s s}(j+1), x_{u}(j+1), x_{u u}(j)\right)=T\left(x_{s s}(j), x_{u}(j), x_{u u}(j+1)\right),
$$

for some map $T$, with the following asymptotics:

$$
\begin{aligned}
x_{s s}(j+1) & =\mathcal{O}\left(x_{u}(j)^{\beta+\omega}\right), \\
x_{u}(j+1) & =\varphi x_{u}(j)^{\beta}+\mathcal{O}\left(x_{u}(j)^{\beta+\omega}\right), \\
x_{u u}(j) & =\mathcal{O}\left(x_{u}(j+1)^{1+\omega}\right),
\end{aligned}
$$

where $\varphi$ is a constant.

Proof. There are smooth vector bundles $F^{s s}$ and $F^{s s, s, u}$ along the stable manifold $W^{s s, s}(p)$, invariant for the flow of $D X$, with $F_{p}^{s s, s, u}=E^{s s} \oplus E^{s} \oplus E^{u}$ and $F_{p}^{s s}=$ $E^{s s}$. Similarly, there are smooth vector bundles $F^{s s}$ and $F^{s s, s, u}$ along the unstable manifold $W^{u, u u}(p)$, with $F_{p}^{s, u, u u}=E^{s} \oplus E^{u} \oplus E^{u u}$ and $F_{p}^{u u}=E^{u u}$. By changing the time parameterization of the flow of $X$, we may assume that leaves $F_{x}^{s s}$ and $F_{x}^{u u}$ are contained in $\Sigma$ for $x \in \Sigma$.

By the transversality assumptions (30), (31), $F^{s s}, F^{u u}, F^{s s, s, u}$ and $F^{s, u, u u}$ extend to continuous vector bundles along $\{p\} \cup \Gamma$; see e.g. Hom96]. The intersection of $F^{s s, s, u}$ and $F^{s, u, u u}$ provides a continuous bundle $F^{s, u}$ along $\Gamma$ with $F_{p}^{s, u}=E^{s} \oplus E^{u}$. Observe that $T_{\Gamma} M=F^{s s} \oplus F^{s, u} \oplus F^{u u}$. It follows that coordinates $\left(x_{s s}, x_{u}, x_{u u}\right)$ on $\Sigma$ satisfying (32)- 35 exist.

Recall that $x_{s s}^{i n}, x_{u}^{i n}, x_{u u}^{i n}$ are coordinates on $\Sigma^{i n}$. If $x_{s s}, x_{u}, x_{u u}$ denote coordinates on $\Sigma$, then

$$
\left(x_{s s}^{i n}, x_{u}^{i n}, x_{u u}^{i n}\right)=\chi^{i n}\left(x_{s s}, x_{u}, x_{u u}\right)
$$

for some smooth local diffeomorphism $\chi^{i n}$. If the coordinates $\left(x_{s s}, x_{u}, x_{u u}\right)$ are chosen so that $\Gamma$ intersects $\Sigma$ in $(0,0,0)$ and $W^{s s, s}=\left\{x_{u, u u}=0\right\}, T_{W^{s s, s}} W^{s s, s, u}=$ 
$\left\{x_{u u}=0\right\}$, then

$$
D \chi^{i n}(0,0,0)=\left(\begin{array}{ccc}
* & * & * \\
0 & * & * \\
0 & 0 & *
\end{array}\right)
$$

and

$$
\chi^{i n}\left(x_{s s}, x_{u}, x_{u u}\right)=D \chi^{i n}(0,0,0)\left(\begin{array}{c}
x_{s s} \\
x_{u} \\
x_{u u}
\end{array}\right)+\left(\begin{array}{c}
\mathcal{O}\left(\|x\|^{2}\right) \\
\mathcal{O}\left(\left\|x_{u, u u}\right\|\|x\|\right) \\
\mathcal{O}\left(\left\|x_{u u}\right\|\|x\|+\left|x_{u}\right|^{2}\right)
\end{array}\right) .
$$

By the implicit function theorem there is a map $\psi$ (defined on a small neighborhood) so that

$$
\left(x_{s s}^{i n}, x_{u}^{i n}, x_{u u}\right)=\psi\left(x_{s s}, x_{u}, x_{u u}^{i n}\right)
$$

and

$$
D \psi(0,0,0)=\left(\begin{array}{ccc}
* & * & * \\
0 & * & * \\
0 & 0 & *
\end{array}\right)
$$

This yields

$$
\left(\begin{array}{c}
x_{s s}^{i n} \\
x_{u}^{i n} \\
x_{u u}
\end{array}\right)=D \psi(0,0,0)\left(\begin{array}{c}
x_{s s} \\
x_{u} \\
x_{u u}^{i n}
\end{array}\right)+\left(\begin{array}{c}
\mathcal{O}\left(\left\|\left(x_{s s}, x_{u}, x_{u u}^{i n}\right)\right\|^{2}\right) \\
\mathcal{O}\left(\left\|\left(x_{u}, x_{u u}^{i n}\right)\right\|\left\|\left(x_{s s}, x_{u}, x_{u u}^{i n}\right)\right\|\right) \\
\mathcal{O}\left(\left\|x_{u u}^{i n}\right\|\left\|\left(x_{s s}, x_{u}, x_{u u}^{i n}\right)\right\|+\left|x_{u}\right|^{2}\right)
\end{array}\right) .
$$

By combining this and the asymptotics for $S$ in Proposition A.2 one solves ( $x_{s s}^{\text {out }}$, $\left.x_{s}^{\text {out }}, x_{u u}\right)$ as functions of $\left(x_{s s}, x_{u}, x_{u u}^{o u t}\right)$. This goes as follows. From the list of equations (40)-(45) below, use equations (42), (43), (44) to solve $x^{i n}=\left(x_{s s}^{i n}, x_{u}^{i n}, x_{u u}^{i n}\right)$ as functions of $\left(x_{s s}, x_{u}, x_{u u}^{\text {out }}\right)$ by the implicit function theorem. Put this in the remaining equations (40), (41), (45).

$$
\begin{aligned}
x_{s s}^{\text {out }} & =S^{s s}\left(x_{s s}^{\text {in }}, x_{u}^{\text {in }}, x_{u u}^{\text {out }}\right), \\
x_{s}^{\text {out }} & =S^{s}\left(x_{s s}^{\text {in }}, x_{u}^{\text {in }}, x_{u u}^{\text {out }}\right), \\
x_{u u}^{\text {in }} & =S^{u u}\left(x_{s s}^{\text {in }}, x_{u}^{\text {in }}, x_{u u}^{\text {out }}\right), \\
x_{s s}^{\text {in }} & =\psi^{s s}\left(x_{s s}, x_{u}, x_{u u}^{\text {in }}\right), \\
x_{u}^{\text {in }} & =\psi^{u}\left(x_{s s}, x_{u}, x_{u u}^{\text {in }}\right), \\
x_{u u} & =\psi^{u u}\left(x_{s s}, x_{u}, x_{u u}^{\text {in }}\right) .
\end{aligned}
$$

Similarly, one relates the coordinates $x_{s s}^{\text {out }}, x_{s}^{\text {out }}, x_{u u}^{\text {out }}$ on $\Sigma^{\text {out }}$ to coordinates on $\Sigma$ by a local diffeomorphism $\chi^{\text {out }}$. The asymptotics are now implied by Proposition A.2

In Section 5 an unfolding of a bellows consisting of non-symmetric homoclinic orbits is considered. To enable treatment of this unfolding, the above constructions must be carried out for families of vector fields. We will indicate how this is done. Let $\left\{X_{\mu}\right\}$ be a smooth one-parameter family of vector fields with $X_{0}=X$.

Notation A.5. Let $(x, y) \mapsto f_{\mu}(x, y)$ with $x \in \mathbb{R}$ be a function that depends smoothly on $y$, on the parameter $\mu$, and on $x$ for $x>0$. When writing $f_{\mu}(x, y)=\mathcal{O}\left(x^{\alpha}\right)$, we will assume that $\left\|D_{y, \mu}^{k} D_{x}^{l} f_{\mu}(x, y)\right\| \leq C_{k+l} x^{\alpha-l}$ for positive constants $C_{k+l}$. 
Theorem A.6. Let $\Psi_{\mu}: \Sigma \rightarrow \Sigma$ be the first return map given by the flow of $X_{\mu}$, and let points $x(j), x(j+1)$ in $\Sigma$ be such that $x(j+1)=\Psi_{\mu}(x(j))$. If (30), (31) holds, then a parameter dependent coordinate system $x=\left(x_{s s}, x_{u}, x_{u u}\right)$ on $\Sigma$ can be chosen so that

$$
\begin{array}{cll}
W^{s s, s}(p) \cap \Sigma= & \left\{x_{u, u u}=0\right\}, \\
W^{u, u u}(p) \cap \Sigma= & \left\{x_{s s, u}=0\right\}, \\
W^{s s, s, u}(p) \cap \Sigma & \bar{\cap}_{W^{s s, s}(p) \cap \Sigma} & \left\{x_{u u}=0\right\}, \\
W^{s, u, u u}(p) \cap \Sigma & \bar{\cap}_{W^{u, u u}(p) \cap \Sigma} & \left\{x_{s s}=0\right\} .
\end{array}
$$

In these coordinates, $x(j+1)$ and $\Psi_{\mu}(x(j))$ are related by

$$
\left(x_{s s}(j+1), x_{u}(j+1), x_{u u}(j)\right)=T_{\mu}\left(x_{s s}(j), x_{u}(j), x_{u u}(j+1)\right),
$$

for some map $T_{\mu}$, with the following asymptotics:

$$
\begin{aligned}
x_{s s}(j+1) & =\mathcal{O}\left(x_{u}(j)^{\beta+\omega}\right), \\
x_{u}(j+1) & =a(\mu)+\varphi(\mu) x_{u}(j)^{\beta}+\mathcal{O}\left(x_{u}(j)^{\beta+\omega}\right), \\
x_{u u}(j) & =\mathcal{O}\left(x_{u}(j+1)^{1+\omega}\right) .
\end{aligned}
$$

Here $a$ and $\varphi$ are smooth functions of $\mu$.

Proof. The normal form given in Lemma A.1 also holds for parameter dependent vector fields, where the linear and higher order terms depend smoothly on the parameter. The corresponding coordinate system depends smoothly on the parameter. The parameter dependent version of Proposition A.2 is proven in much the same way as the proposition itself; one considers, in addition, derivatives with respect to the parameter; see also [Den89, Den96. The choice of coordinates in Theorem A.4 can be made parameter dependent. An additional translation results from breaking the homoclinic connection, yielding the term $a(\mu)$.

We have confined ourselves to first return maps for a single homoclinic orbit, on a single cross-section. Studying homoclinic bellows, consisting of two homoclinic orbits $\Gamma_{1}, \Gamma_{2}$, requires the introduction of two cross-sections $\Sigma_{1}, \Sigma_{2}$, each transverse to one of the homoclinic orbits. One distinguishes four maps $\Psi^{i, j}: \Sigma_{i} \rightarrow \Sigma_{j}$ that together form the return map $\Psi: \Sigma_{1} \cup \Sigma_{2} \rightarrow \Sigma_{1} \cup \Sigma_{2}$. It is clear that for all four maps one can write down asymptotic expansions in Shil'nikov variables as above.

\section{Appendix B. ShIL'Nikov VARIABLes AND Lin's METHOD}

In this appendix we point out the connection between Shil'nikov variables and Lin's method, a method frequently applied to obtain bifurcation equations for global bifurcation problems Lin90, San93, Kno00. We use the notation introduced in the previous section. As pointed out, for each given sufficiently large $\tau$ and given $x_{u u}^{\text {out }}$ and $x_{s s}^{i n}$ there is a unique orbit $\mathfrak{x}=\{x(t), t \in[0, \tau]\}$ such that $x(0) \in \Sigma^{i n}, x_{s s}(0)=$ $x_{s s}^{\text {in }}, x(\tau) \in \Sigma^{\text {out }}, x_{u u}(\tau)=x_{u u}^{\text {out }}$. In other words, $x(\cdot)$ solves the Shil'nikov problem for the Shil'nikov data $\left(\tau,\left(x_{s s}^{i n}, 1\right),\left(1, x_{u u}^{\text {out }}\right)\right)$. With respect to a local Poincaré map $\psi^{l o c}$ the coordinates $\left(x_{s s}^{i n}, x_{u u}^{o u t}, x_{u}^{i n}\right)$ are addressed as Shil'nikov variables. Note that there is a one-to-one correspondence between $\tau$ and $x_{u}^{i n}$. So the coordinates given in Theorem A.4 can be seen as global Shil'nikov variables in a cross-section $\Sigma$ with respect to first return map $\psi$. We denote the return map in Shil'nikov variables by 
$T$. Consider points $x(j), j \in \mathbb{Z}$, in $\Sigma$. The points under consideration lie on the same orbit iff

$$
\left(x_{s s}(j+1), x_{u}(j+1), x_{u u}(j)\right)-T\left(x_{s s}(j), x_{u}(j), x_{u u}(j+1)\right)=0, \quad j \in \mathbb{Z},
$$

or more detailed

$$
\begin{aligned}
x_{s s}(j+1)-\mathcal{O}\left(x_{u}(j)^{\beta+\omega}\right) & =0 \\
x_{u}(j+1)-\varphi x_{u}(j)^{\beta}+\mathcal{O}\left(x_{u}(j)^{\beta+\omega}\right) & =0 \\
x_{u u}(j)-\mathcal{O}\left(x_{u}(j+1)^{1+\omega}\right) & =0 .
\end{aligned}
$$

Let $l_{\mathbb{R}^{k}}^{\infty}$ be the space of bi-infinite sequences of elements of $\mathbb{R}^{k}$ equipped with the supremum norm. Then equation (47) can be considered as an equation in $l_{\mathbb{R}^{q_{s s}}}^{\infty} \times$ $l_{\mathbb{R}}^{\infty} \times l_{\mathbb{R}_{\text {quu }}}^{\infty}$. In the same way the first and third equation in (48) can be seen. The first and third equation of this system can be solved for

$$
\left(x_{s s}(j), x_{u u}(j)\right)_{j \in \mathbb{Z}}\left(\left(x_{u}(i)\right)_{i \in \mathbb{Z}}\right) .
$$

Plugging this into the second equation of (48) we arrive at

$$
x_{u}(j+1)-\varphi x_{u}(j)^{\beta}+\mathcal{O}\left(x_{u}(j)^{\beta+\omega}\right)=0 .
$$

This proves

Theorem B.1. Let $X$ be a vector field possessing a homoclinic orbit $\Gamma$ as introduced in Section $\mathrm{A}$. Further, let $\Sigma$ be a cross-section of $\Gamma$ equipped with Shil'nikov coordinates $\left(x_{s s}, x_{u}, x_{u u}\right)$. Then for each sequence $X_{u}=\left(x_{u}(i)\right)_{i \in \mathbb{Z}}$ with each $x_{u}(i)$ small enough, there exists a unique "discontinuous" orbit $\mathfrak{o}\left(X_{u}\right)$ allowing in $\Sigma$ jumps in the $x_{u}$-direction.

This is one of the core statements of Lin's method [Lin90, San93, Kno00. As mentioned there is a one-to-one correspondence between the revolution time $\tau$ and the coordinate $x_{u}$. Therefore, the statement of Theorem B.1 can be alternatively formulated as follows: for each sequence $\mathbf{T}=(\tau(i))_{i \in \mathbb{Z}}$ with each $\tau(i)$ large enough, there exists a unique "discontinuous" orbit $\mathfrak{o}(\mathbf{T})$ allowing in $\Sigma$ jumps in the $x_{u^{-}}$ direction.

Note that the coordinate surfaces of $x_{s s}$ and $x_{u u}$ are the traces of $W^{s s, s}(p)$ and $W^{u, u u}(p)$, respectively, in $\Sigma$. The coordinate line of $x_{u}$ is transverse to the direct sum of the tangent spaces of $W^{s s, s}(p)$ and $W^{u, u u}(p)$.

\section{Appendix C. Center manifolds}

This appendix contains the proofs of the center manifold result Theorem 2.1 and the version Theorem 2.2 discussing parameter dependence. We will prove the results for the eigenvalue condition $-\lambda^{s}=\lambda^{u}$, since this is satisfied for the reversible and conservative systems we study. This is the more complicated case, and is easily altered into a proof for general eigenvalue conditions. A remark on this point is contained in the proof below. The section concludes with remarks on center manifolds for reversible systems.

Fix a sequence $\eta: \mathbb{Z} \rightarrow\{1,2\}$. Let $x^{\eta}(j+1)=\Psi\left(x^{\eta}(j)\right), j \in \mathbb{Z}$, be an orbit of $\Psi$ contained in the nonwandering set of $\Psi$, with

$$
x^{\eta}(j) \in \Sigma_{\eta(j)} .
$$


Applying Theorem A.4 (compare the remark at the end of Appendix A), for each triple $(\eta(j), \eta(j+1), \eta(j+2))$ there are maps $\left(G_{j+1}^{s s}, G_{j+1}^{u}, G_{j+1}^{u u}\right)$, so that

$$
\begin{aligned}
x_{s s}^{\eta}(j+1) & =G_{j+1}^{s s}\left(x_{s s}^{\eta}(j), x_{u}^{\eta}(j), x_{u u}^{\eta}(j+1)\right), \\
x_{u}^{\eta}(j+1) & =G_{j+1}^{u}\left(x_{s s}^{\eta}(j), x_{u}^{\eta}(j), x_{u u}^{\eta}(j+1)\right), \\
x_{u u}^{\eta}(j) & =G_{j+1}^{u u}\left(x_{s s}^{\eta}(j), x_{u}^{\eta}(j), x_{u u}^{\eta}(j+1)\right) .
\end{aligned}
$$

We suppress the dependence of $G_{j+1}$ on the triple $(\eta(j), \eta(j+1), \eta(j+2))$ from the notation. From (51), (52), (53) it follows that we can write

$$
x^{\eta}(j+1)=\mathcal{G}_{j+1}\left(x^{\eta}\right) .
$$

Note that $\mathcal{G}_{j+1}$ depends only on $x^{\eta}(j), x^{\eta}(j+1), x^{\eta}(j+2)$. The asymptotic expansions given in Theorem A.4 imply that

$$
\begin{aligned}
x_{s s}^{\eta}(j+1) & =\mathcal{O}\left(x_{u}^{\eta}(j)^{1+\omega}\right), \\
x_{u}^{\eta}(j+1) & =\varphi_{\eta(j), \eta(j+1)} x_{u}^{\eta}(j)+\mathcal{O}\left(x_{u}^{\eta}(j)^{1+\omega}\right), \\
x_{u u}^{\eta}(j+1) & =\mathcal{O}\left(x_{u}^{\eta}(j+1)^{1+\omega}\right),
\end{aligned}
$$

where $\varphi_{\eta(j), \eta(j+1)}>0$.

Proof of Theorem 2.1. A center manifold $W_{\eta}^{c}$ is obtained as an intersection of a center stable manifold $W_{\eta}^{s s, c}$, tangent to $E^{s s} \times E^{u}$ at $\Gamma_{\eta(0)}$, and a center unstable manifold $W_{\eta}^{c, u u}$, tangent to $E^{u} \times E^{u u}$ at $\Gamma_{\eta(0)}$. We will discuss the construction of a center stable manifold, the construction of a center unstable manifold proceeds analogous.

Our proof is an adaptation, making use of the Shil'nikov coordinates from Theorem A.4 of existing proofs for center stable manifolds of smooth diffeomorphisms near equilibria in a Euclidean space; see Irw80. For the construction of these local center stable manifolds one extends the diffeomorphism near the equilibrium to a map globally close to the linearized map at the equilibrium. We will proceed similarly and extend $\mathcal{G}_{j+1}$ to $\mathbb{R}^{q_{s s}} \times \mathbb{R} \times \mathbb{R}^{q_{u u}}$. The maps $\mathcal{G}_{j+1}$ are defined for $x_{u}^{\eta}$ positive, and then for $x_{u}^{\eta}$ nonnegative by continuity. Replacing the expressions (51), (52), (53) by

$$
\begin{aligned}
x_{s s}^{\eta}(j+1) & =G_{j+1}^{s s}\left(x_{s s}^{\eta}(j),\left|x_{u}^{\eta}(j)\right|, x_{u u}^{\eta}(j+1)\right), \\
x_{u}^{\eta}(j+1) & =\operatorname{sign}\left(x_{u}^{\eta}(j)\right) G_{j+1}^{u}\left(x_{s s}^{\eta}(j),\left|x_{u}^{\eta}(j)\right|, x_{u u}^{\eta}(j+1)\right), \\
x_{u u}^{\eta}(j+1) & =G_{j+1}^{u u}\left(x_{s s}^{\eta}(j+1),\left|x_{u}^{\eta}(j+1)\right|, x_{u u}^{\eta}(j+2)\right),
\end{aligned}
$$

$\mathcal{G}_{j+1}$ becomes defined for all small values of $x_{u}^{\eta}$. The asymptotic expansions are valid for all small values of $x_{u}^{\eta}$, so that $\mathcal{G}_{j+1}$ is continuously differentiable and

$$
D \mathcal{G}_{j+1}(\mathbf{0})\left(x^{\eta}\right)=\left\{\left(0, \varphi_{\eta(j), \eta(j+1)} x_{u}^{\eta}(j), 0\right)\right\} .
$$

Let $\phi$ be a smooth nonnegative cut-off function on $\mathbb{R}^{n-1}: \phi \equiv 1$ on the ball of radius 1 around the origin and $\phi \equiv 0$ outside the ball of radius 2 around the origin. Write $\phi_{\epsilon}(x)=\phi(x / \epsilon)$. For some small positive value of $\epsilon$, let

$$
\mathcal{H}_{j+1}=\phi_{\epsilon} \mathcal{G}_{j+1}+\left(1-\phi_{\epsilon}\right) D \mathcal{G}_{j+1}(0,0,0) .
$$

Observe that $\mathcal{H}_{j+1}$ is globally $C^{1}$ close to $D \mathcal{G}_{j+1}(\mathbf{0})$ and equals $\mathcal{G}_{j+1}$ near the origin. For fixed $\left(x_{s s}, x_{u}\right) \in \mathbb{R}^{q_{s s}} \times \mathbb{R}$, denote by $\mathcal{H}$ the map given by

$$
\mathcal{H}(y)(k)= \begin{cases}\left(x_{s s}, x_{u}, \mathcal{H}_{k}^{u u}\left(x_{s s}, x_{u}, y_{u u}(1)\right),\right. & \text { if } k=0 \\ \mathcal{H}_{k}(y), & \text { if } k>0\end{cases}
$$


Note that we suppress the dependence of $\mathcal{H}$ on $\left(x_{s s}, x_{u}\right)$ from the notation.

Write $\mathcal{C}_{\alpha}$ for the set of sequences $\mathbb{N} \rightarrow \mathbb{R}^{n-1}$, indexed by the nonnegative integers $\mathbb{N}$, equipped with the norm

$$
\|x\|_{\alpha}=\sup _{k \in \mathbb{N}} \alpha^{k}\|x(k)\|
$$

Let $A: \mathcal{C}_{1} \rightarrow \mathcal{C}_{\alpha}$ be given by

$$
A x(k)=\alpha^{-k} x(k)
$$

Fix $\alpha>1$ and define

$$
\mathcal{J}=A^{-1} \circ \mathcal{H} \circ A
$$

Note that $\mathcal{J}$ maps $\mathcal{C}_{1}$ into $\mathcal{C}_{1}$. Since $\mathcal{H}_{j}$ is close to $D \mathcal{G}_{j}(0,0,0)$, for some $\alpha>1$, $\mathcal{J}$ is a contraction on $\mathcal{C}_{1}$. (We remark that, if $\beta>1, \mathcal{H}$ is a contraction and the introduction of the map $A$ is not needed. The case $\beta<1$ can be brought to $\beta>1$ by reversing the direction of time.) The map $\mathcal{J}$ therefore possesses a unique fixed point. By uniqueness, the fixed point $\zeta$ of $\mathcal{J}$ satisfies

$$
\zeta(k+1)=\alpha^{-(k+1)} \Psi\left(\alpha^{k} \zeta(k)\right),
$$

if these points lie in $\Sigma_{1} \cup \Sigma_{2}$ and have positive $x_{u}$ coordinates. Thus $\alpha^{k} \zeta(k)$ is an orbit for $\Psi$ for as long as it is in $\Sigma_{1} \cup \Sigma_{2}$ and has positive $x_{u}$ coordinates. Write $w\left(x_{s s}, x_{u}\right)=\zeta(0)$ and define

$$
W_{\eta}^{s s, c}=\bigcup_{\left(x_{s s}, x_{u}\right)} w\left(x_{s s}, x_{u}\right) .
$$

It must be kept in mind that the constructions for negative or large values of $x_{u}$ bear no relevance to the actual vector field.

Since $\mathcal{J}$ depends continuously on $\left(x_{s s}, x_{u}\right),\left(x_{s s}, x_{u}\right) \mapsto w\left(x_{s s}, x_{u}\right)$ is continuous. We claim that $w$ is continuously differentiable. As shown above, $\mathcal{J}$ is a contraction on $\mathcal{C}_{1}$. Equivalently, $\mathcal{H}$ is a contraction on $\mathcal{C}_{\alpha}$. Since $\mathcal{G}$ is not differentiable Irw80, GilVan87, the implicit mapping theorem cannot be applied to demonstrate smoothness of $w$. It follows from Theorem 2 in GilVan87 that $w$ is indeed $C^{1}$.

It remains to see that $w$ depends continuously on $\eta$. If $l(j)=\eta(j)$ for $-N \leq$ $j \leq N$, then the maps $\mathcal{G}_{j}$ for the symbolic sequences $\eta$ and $l$ equal each other for $-N+1 \leq j \leq N-1$. It follows that the fixed points lie close if $N$ is large, hence that $w$ depends continuously on $\eta$.

Non-symmetric homoclinic orbits in reversible systems break under perturbations of the vector field. To treat this bifurcation problem, the construction of center manifolds for families of vector fields must be considered. Theorem 2.2 establishes persistence of the center manifolds from Theorem 2.1.

Proof of Theorem 2.2. Write, as for single vector fields, now applying Theorem A.6.

$$
x^{\eta}(j+1)=\mathcal{G}_{j+1}\left(x^{\eta}\right) .
$$

The asymptotic expansions given in Theorem A.6 imply that

$$
\begin{aligned}
x_{s s}^{\eta}(j+1) & =\mathcal{O}\left(x_{u}^{\eta}(j)^{1+\omega}\right), \\
x_{u}^{\eta}(j+1) & =a_{\eta(j), \eta(j+1)}+\varphi_{\eta(j), \eta(j+1)} x_{u}^{\eta}(j)+\mathcal{O}\left(x_{u}^{\eta}(j)^{1+\omega}\right), \\
x_{u u}^{\eta}(j+1) & =\mathcal{O}\left(x_{u}^{\eta}(j+1)^{1+\omega}\right),
\end{aligned}
$$

where $a_{\eta(j), \eta(j+1)}, \varphi_{\eta(j), \eta(j+1)}$ depend smoothly on the parameter $\mu$ and satisfy $a_{\eta(j), \eta(j+1)}(0)=0, \varphi_{\eta(j), \eta(j+1)}>0$. The construction proceeds as above, in the 
proof of Theorem 2.1. yielding parameter dependent center stable manifolds $W_{\eta}^{s s, c}$ for small values of $\mu$,

$$
W_{\eta}^{s s, c}=\bigcup_{\left(x_{s s}, x_{u}\right)} w_{\mu}\left(x_{s s}, x_{u}\right) .
$$

Center manifolds are again obtained as transverse intersections of center stable and center unstable manifolds. Theorem 2 in GilVan87 implies that $w$ is $C^{1}$ jointly in $\left(x_{s s}, x_{u}\right)$ and $\mu$.

Let $w_{\mu}$ be the map whose graph yields $W_{\eta}^{s s, c}$ as in the proof above. It is straightforward to provide bounds for $\left\|D w_{\mu}\right\|$. The proof of Theorem 3 in GilVan87, shows how to bound the Lipschitz constants, and thus the derivatives, of $w_{\mu}$. One concludes that $\left\|D w_{\mu}\right\|$ is bounded, uniformly in $\mu$ and $\eta$.

For reversible systems on $2 n$-dimensional manifolds the involution should map center manifolds to center manifolds. Given is an involution $R$ on $\Sigma_{1} \cup \Sigma_{2}$ and a first return map $\Psi$ which is reversible with respect to $R$. Also given is an involution $\mathcal{R}$ on $\mathcal{B}_{2}$, connected to $R$ by $\Upsilon(R x)=\mathcal{R} \Upsilon(x)$, for each $\Psi$-orbit $x$. We claim that center manifolds $W_{\eta}^{c}$ can be constructed so that

$$
W_{\mathcal{R} \eta}^{c}=R W_{\eta}^{c} \text {. }
$$

To see this, choose symmetric cut-off functions in the above constructions. In the coordinate system $x=\left(x_{s s}, x_{u}, x_{u u}\right)$, the involution $R$ is given by

$$
R\left(x_{s s}, x_{u}, x_{u u}\right)=\left(x_{u u}, x_{u}, x_{s s}\right) .
$$

Let $G_{j+1}=\left(G_{j+1}^{s s}, G_{j+1}^{u}, G_{j+1}^{u u}\right)$ be the Shil'nikov map given in (51), (52), (53). Note that $G_{j+1}$ is symmetric,

$$
G_{j+1} R=R G_{j+1} .
$$

In order to establish a symmetry for the mapping $\mathcal{J}$ we have to distinguish the $\mathcal{J}$ associated with the center stable manifold from that one which belongs to the center unstable manifold. We denote them by $\mathcal{J}^{s s, c}$ and $\mathcal{J}^{c, u u}$, respectively. Likewise, all quantities arising in the proof of Theorem 2.1 can be labeled with the superscripts "ss, $c$ " or " $c$, uu" respectively. Note that in this notation $\mathcal{C}_{\alpha}^{c, u u}$ is the set of sequences $-\mathbb{N} \rightarrow \mathbb{R}^{2 n-1}$. Also, we include in the notation the dependence on the itinerary $\eta$ and the Shil'nikov variables $\left(x_{s s}, x_{u}\right)$ and $\left(x_{u}, x_{u u}\right)$, respectively. So we can conceive $\mathcal{J}^{s s, c}$ as a mapping $\mathcal{B}_{2} \times \mathbb{R}^{n-1} \times \mathbb{R} \times \mathcal{C}_{1}^{s s, c} \rightarrow \mathcal{C}_{1}^{s, c}$. Further, define $\hat{R}: \mathcal{C}_{1}^{s s, c} \rightarrow \mathcal{C}_{1}^{c, u u}$ by

$$
((\hat{R} x)(-k))_{k \in \mathbb{N}}=(R x(k))_{k \in \mathbb{N}} .
$$

Working through the proof of Theorem 2.1 we end up with

$$
\mathcal{J}^{s s, c}\left(\eta, x_{s s}, x_{u}, \zeta\right)=\hat{R} \mathcal{J}^{c, u u}\left(\mathcal{R} \eta, x_{u}, x_{u u}, \hat{R} \zeta\right) .
$$

This provides the assertion on the symmetry of the center manifolds.

\section{REFERENCES}

[AroGolKru91] D.G. Aronson, M. Golubitsky, M. Krupa, Coupled arrays of Josephson junctions and bifurcation of maps with $S_{N}$ symmetry, Nonlinearity 4, 861-902 (1991). MR.1124338 (93d:58107a)

[ChaGro97] A. R. Champneys, M.D. Groves, A global investigation of solitairy wave solutions to a two-parameter model for water waves, J. Fluid Mech. 342, 199-229 (1997). MR1460660 (98f:76015)

[ChoLiuYi00] S.-N. Chow, W. Liu, Y. Yi, Center manifolds for invariant sets, J. Differential Equations 168 (2000), 355-385. MR1808454(2002a:37028) 
[Den89] B. Deng, The Shil'nikov problem, exponential expansion, strong $\lambda$-lemma, $C^{1}$ linearization, and homoclinic bifurcation, Journ. Diff. Eq. 79, 189-231 (1989). MR:1000687 (90k:58161)

[Den89b] B. Deng, Exponential expansion with Šil'nikov's saddle-focus, Journ. Diff. Eq. 82, 156-173 (1989). MR1023305 (91b:58183)

[Den93] B. Deng, Homoclinic twisting bifurcations and cusp horseshoe maps, J. Dynam. Differential Equations 5 (1993), 417-467. MR.1235038(94i:58134)

[Den96] B. Deng, Exponential expansion with principal eigenvalues. Nonlinear dynamics, bifurcations and chaotic behavior, Internat. J. Bifur. Chaos Appl. Sci. Engrg. 6, 1161-1167 (1996). MR:1409417 (97g:58123)

[Dev76] R.L. Devaney, Reversible diffeomorphisms and flows, Trans. Amer. Math. Soc. 218, 89-113 (1976). MR0402815 (53:6629)

[Dev77] R.L. Devaney, Blue sky catastrophes in reversible and Hamiltonian systems, Indiana Univ. Math. Journal 26, 247-263 (1977). MR0431274 (55:4275)

[Dev58] R. DeVogelaere, On the structure of symmetric periodic solutions of conservative systems, with applications, in: Contributions to the theory of nonlinear oscillations, vol. IV, Annals of Mathematics Studies no. 41, Princeton Univ. Press. 1958. MR0101362 (21:174)

[FieTur96] B. Fiedler, D. Turaev, Coalescence of reversible homoclinic orbits causes elliptic resonance, Internat. J. Bifur. Chaos Appl. Sci. Engrg. 6, 1007-1027 (1996). MR1409408 (97i:58121)

[GilVan87] S.A. van Gils, A. Vanderbauwhede, Center manifolds and contractions on a scale of Banach spaces, J. of Functional Analysis 72, 209-224 (1987). MR0886811 (88d:58085)

[Hom93] A.J. Homburg, Some global aspects of homoclinic bifurcations of vector fields, PhD-thesis, University of Groningen, 1993.

[Hom96] A.J. Homburg, Global aspects of homoclinic bifurcations of vector fields, Memoirs of the A.M.S. 578, (1996). MR1327210(96i:58125)

[Hom00] A.J. Homburg, Singular heteroclinic cycles, Journ. Diff. Eq. 161, 358-402 (2000). MR 1744144 (2001a:37030)

[HomKokKru94] A.J. Homburg, H. Kokubu, M. Krupa, The cusp horseshoe and its bifurcations in the unfolding of an inclination flip homoclinic orbit, Ergod. Th. \& Dynam. Sys. 14, 667-693 (1994). MR1304138 (96a:58134)

[Irw80] M.C. Irwin, A new proof of the pseudostable manifold theorem, J. London Math. Soc. 21, 557-566 (1980). MR0577730 (81h:34051)

[KisKokOka93] M. Kisaka, H. Kokubu, H. Oka, Bifurcations to $N$-homoclinic orbits and $N$ periodic orbits in vector fields, J. Dyn. Diff. Eq. 5, 305-357 (1993). MR 1223451 (94m:58158)

[KisKokOka93b] M. Kisaka, H. Kokubu, H. Oka, Supplement to homoclinic doubling bifurcation in vector fields. In: Dynamical Systems, Longman, 1993. MR1213943 (94c:58136)

[Kno97] J. Knobloch, Bifurcation of degenerate homoclinics in reversible and conservative systems, J. Dyn. Diff. Eq. 9, 427-444 (1997). MR1464081 (99f:58147)

[Kno00] J. Knobloch, Lin's method for discrete dynamical systems, J. of Difference Eqn. and Appl. 6, 577-623 (2000). MR1802448(2001k:37018)

[KolLer96] O.Yu. Koltsova, L.M. Lerman, Families of transverse Poincaré homoclinic orbits in $2 N$-dimensional Hamiltonian systems close the system with a loop to a saddle -center, Internat. J. Bifur. Chaos Appl. Sci. Engrg. 6, 991-1006 (1996). MR1409407 (97g:58060)

[Lin90] X.-B. Lin, Using Melnikovs method to solve Shilnikovs problems, Proc. Roy. Soc. Edinburgh 116A, 295-325 (1990). MR1084736(92b:58195)

[MorHed38] M. Morse, G.A. Hedlund, Symbolic dynamics, Amer. J. Math. 60, 815-866 (1938).

[OvsShi87] I.M. Ovsyannikov, L.P. Shil'nikov, On systems with saddle-focus homoclinic curve, Math. USSR Sbornik, 58, 557-574 (1987).

[RouRou96] R. Roussarie, C. Rousseau, Almost planar homoclinic loops in $\mathbb{R}^{3}$, J. Differential Equations 126 (1996), 1-47. MR1382055 (97e:58170) 
[San93] B. Sandstede, Verzweigungstheorie homokliner Verdopplungen, PhD-thesis, Free University of Berlin, 1993.

[San00] B. Sandstede, Center manifolds for homoclinic solutions, J. Dyn. Diff. Eq. 12, 449-510 (2000). MR 1800130 (2001m:37167)

[SanJonAle97] B. Sandstede, C.K.R.T. Jones, J.C. Alexander, Existence and stability of Npulses on optical fibres with phase-sensitive amplifiers, Physica D 106, 167-206 (1997). MR 1460453 (98h:35227)

[ShaTur99] M.V. Shashkov, D.V. Turaev, An existence theorem of smooth nonlocal center manifolds for systems close to a system with a homoclinic loop, J. Nonlinear Sci. 9, 525-573 (1999). MR.1707981 (2000f:37026)

[Shi65] L.P. Shil'nikov, A case of the existence of a countable number of periodic motions, Soviet Math. Dokl. 6, 163-166 (1965).

[Shi67] L.P. Shil'nikov, On the Poincaré-Birkhoff problem, Math. USSR Sbornik 3, 353371 (1967)

[Tur88] D.V. Turaev, Bifurcations of a homoclinic "figure eight" of a multidimensional saddle, Russian Math. Surveys 43, 264-265 (1988). MR0971495 (90m:58156)

[Tur01] D.V. Turaev, Multi-pulse homoclinic loops in systems with a smooth first integral, in: Ergodic theory, analysis, and efficient simulation of dynamical systems, Springer Verlag, 2001. MR.1850326 (2002f:37094)

[TurShi87] D.V. Turaev, L.P. Shil'nikov, On bifurcations of a homoclinic "figure eight" for a saddle with negative saddle value, Soviet. Math. Dokl. 34, 397-401 (1987).

[Van92] A. Vanderbauwhede, Bifurcation of degenerate homoclinics, Results in Mathematics 21, 211-223 (1992). MR1146644 (93a:58131)

[VanFie92] A. Vanderbauwhede, B. Fiedler, Homoclinic period blow-up in reversible and conservative systems, Z. Angew. Math. Phys. 43, 292-318 (1992). MR1162729 (93f:58209)

[WagCha02] T. Wagenknecht, A.R. Champneys, When gap solitons become embedded solitons: a generic unfolding, Phys. D 177 (2003), 50-70. MR.1965327|(2004a:37103)

[Yan87] E. Yanagida, Branching from double pulse solutions from single pulse solutions in nerve axon equations, J. Differential Equations 66 (1987), 243-262. MR0871997 $(88 \mathrm{c}: 35078)$

KdV Institute for Mathematics, University of Amsterdam, Plantage Muidergracht 24, 1018 TV Amsterdam, The Netherlands

E-mail address: alejan@science.uva.nl

Institute for Mathematics, Technical University Ilmenau, Postbox 1005 65, D-98684 Ilmenau, Germany

E-mail address: knobi@mathematik.tu-ilmenau.de 